\title{
A Uniform Theory of CONDitionals
}

\author{
William B. StarR \\ Sage School of Philosophy, Cornell University \\ 218 Goldwin Smith Hall \\ Ithaca, NY 14850
}

\begin{abstract}
A uniform theory of conditionals is one which compositionally captures the behavior of both indicative and subjunctive conditionals without positing ambiguities. This paper raises new problems for the closest thing to a uniform analysis in the literature (Stalnaker 1975) and develops a new theory which solves them. I also show that this new analysis provides an improved treatment of three much-discussed phenomena (the import-export equivalence, reverse Sobel-sequences and disjunctive antecedents). While these results concern central issues in the study of conditionals, broader themes in the philosophy of language and formal semantics are also engaged here. This new analysis exploits a dynamic conception of meaning where the meaning of a symbol is its potential to change an agent's mental state (or the state of a conversation) rather than being the symbol's content (e.g. the proposition it expresses). The analysis of conditionals is also built on the idea that the contrast between subjunctive and indicative conditionals parallels a contrast between revising and consistently extending some body of information.
\end{abstract}

Key words: Conditionals, Dynamic Semantics, Meaning, Logic

\section{Introduction}

English conditionals can be distinguished into two basic classes. Some, like (2), mix past tense marking in the antecedent with a modal in the consequent. Others, like (1), lack this combination. ${ }^{1}$

Email: will.starr@cornell.edu.

$U R L:$ http://williamstarr.net.

1 The indicative and subjunctive labels are flawed, but ubiquitous. (1) and (2) are not meant to be exhaustive, but rather paradigmatic lists. I will not propose an analysis of habitual (Bob dances if Leland dances) or future (Bob will dance if Leland dances) indicatives. These require an independent analysis of habituals and future tense, which are more complex than simple past and present tenses, with a 
(1) a. If Bob danced, Leland danced

('Indicative')

b. If Bob is dancing, Leland is dancing

(2) a. If Bob had danced, Leland would have danced

('Subjunctive')

b. If Bob had danced, Leland might have danced

c. If Bob had danced, Leland could have danced

d. If Bob danced, Leland would dance

e. If Bob were to dance, Leland would dance

This seemingly subtle linguistic difference allows these two kinds of conditionals to be used differently. Only subjunctives can be used counterfactually: (2a)-(2e), unlike (1a) and (1b), can be felicitously used even when it is mutual information among the conversationalists that Bob never danced and never will. This difference appears to be a basic one. It survives even in languages which lack tense morphology. For example, the tenseless language Kalaallisut forms all conditionals using hypothetical subordinate mood in the antecedent but in counterfactuals includes an additional particle in both clauses expressing 'remote modality' (Bittner 2011). Further subdivisions exist and require explanation, but it is this basic distinction that is investigated here.

Any semantic theory of conditionals must ultimately deliver a uniform analysis of the two species. That is, it must ascribe the same meanings to the constituents they share, e.g. if, and derive their semantic commonalities from these meanings. Further, it must explain their semantic differences in terms of the meaning of the constituents they do not share. This is just to accept a minimal version of compositionality and the fact that the two species share constituents which are not mere homonyms. ${ }^{2}$ Despite the immense attention conditionals have received, there theory that comes the closest to delivering this result: Stalnaker $(1968,1975)$. In this paper I raise three problems for this attempt at uniformity and propose a new uniform theory that solves them. This improved theory builds on the analysis of indicative conditionals developed in Starr (2013a) and Gillies (2009), which already has important advantages over Stalnaker's (1975). I will also show that the compositional ex-

plausible analysis of conditionals. Kaufmann (2005) takes up this project.

2 The cross-linguistic distribution of conditional particles indicates that they are not homonymous. Another obvious but unappreciated mark against homonymy was pointed out to me by Tom Donaldson: there isn't a subjunctive reading of (1) or an indicative reading of $(2)$.

W. StarR

$03 / 23 / 13$ 
tension to subjunctives proposed here offers analyses of three phenomena that are problematic for Stalnaker (1968) and Lewis (1973): the validity of importexport, disjunctive antecedents (Fine 1975) and reverse Sobel sequences (von Fintel 2001; Gillies 2007) ( $§ 4$ introduces these phenomena). There is, however, a broader narrative here that connects three more general themes in the philosophy of language.

The prominent semantic difference between indicative and subjunctive conditionals seems to parallel two different doxastic processes: a consistent extension versus a revision of an agent's beliefs. ${ }^{3}$ This parallel is a promising way to align work on the semantics of conditionals with work in epistemology and the philosophy of science on inference and inquiry. ${ }^{4}$ Why seek such alignment? Leibniz, Boole, Frege and others established the idea of language as an epistemic tool, and conditionals inhabit a special place in this conception. One can infer $B$ from $A$ and $B$ if $A$. So whatever accepting a conditional amounts to, it should at least cohere with a rational policy for inferring $B$ from $A$. Extending this correspondence to natural language seems appropriate, since the basic inference of modus ponens is as central as there is in any formal language. It therefore seems not only appropriate to connect our studies of natural language conditionals and our theories of belief change, it seems necessary to explain how natural language does what it does.

Connecting belief change and conditionals leads to a yet more difficult question. Subjunctive conditionals are the standard medium for stating and reasoning about a certain kind of dependence in the world around us, e.g. if the light switch were up, the light would be on. But what is it about the world (and us) that makes this capacity so powerful? As Goodman (1947) detailed, the question of what it takes to make a subjunctive conditional true is difficult enough, without also saying why these kinds of truths are particularly powerful. The Lewis-Stalnaker semantics intentionally avoids Goodman's question and focuses instead on a logical question (Stalnaker 1968: §I): what patterns of inference govern subjunctive conditionals? They showed that it is possible to state constraints on subjunctive truth-conditions that are strong enough to

3 Many authors have explored this idea. See van Rooy (2006: §5.6).

4 The latter work draws a distinction between imaging and conditionalization (e.g. Lewis 1975; Gärdenfors 1982) or belief revision and belief update (e.g. Katsuno \& Mendelzon 1992).

W. STARR

$03 / 23 / 13$ 
make interesting predictions about their logical behavior, but too weak to engage Goodman's difficulties. ${ }^{5}$ This feature of the theory can be obscured when Lewis and Stalnaker say that the truth of a subjunctive conditional depends on the relative similarity of different possible worlds. But the constraints on similarity they require are far less demanding than ordinary similarity, as they have mentioned in response to critics. ${ }^{6}$ So while their logical constraints happen to partially characterize ordinary similarity, they make no claim that ordinary similarity is actually involved in the truth or evaluation of subjunctive conditionals. Filling out an account of similarity or even constructing the similarity function needed to make a particular conditional true confronts what they are trying to avoid: Goodman's problems (Thomason 2007). Their claim is that a relation similar to similarity captures the logic of subjunctive conditionals. It certainly seems possible to contend that this conservativism mitigates the explanatory depth of their semantics, but it should be uncontroversial that Goodman's question remains a deep and unanswered one.

I wish to join Thomason (2007:2) in expressing optimism that new methods from artificial intelligence will help with Goodman's problems. This is no accident, since Goodman's problems parallel one of AI's signature puzzles: the frame problem. ${ }^{7}$ In my opinion, Pearl's (2000) work on causal modeling is the most suitable for developing a semantics of subjunctive conditionals. While I pursue that project elsewhere, it is relevant to this paper as well. Any semantics built on the causal modeling approach will be variably-strict and many authors have objected that this gives the wrong logic for subjunctive conditionals. These arguments were those mentioned earlier concerning the validity of import-export, disjunctive antecedents and reverse Sobel sequences. Since the theory developed here addresses these arguments while giving a variably-

$\overline{5}$ These constraints are detailed in Definition 16, Appendix A.1.

6 E.g. Stalnaker (1984: Ch.7) and Lewis (1979a).

7 Consider a robot with a model of its environment who can perform actions which change that environment. How does the robot decide what has changed and what hasn't? (McCarthy \& Hayes 1969) Striking the match usually leads to fire. But sometimes not. The match could be wet, the striking surface could be too smooth or there could be too little oxygen or too much wind, or the robot could have confused a twig for a match. Where does this list end? The robot will also need a list of the things that don't change! Goodman observed: it seems to be true that if the match had been struck, it would have lit. But it doesn't light under any number of conditions and listing them without circularity is non-trivial. See Shanahan (2009).

W. STARR

$03 / 23 / 13$ 
strict analysis of subjunctives, it is an important piece of a theory which meets Goodman's problems head on.

The third theme in the philosophy of language relevant here is a particular debate about the nature of linguistic meaning. Everyone agrees that as a conversation unfolds the conversationalists' information changes. But there is a debate about how our knowledge of linguistic meaning (semantics) and general purpose rationality (pragmatics) conspire to make this happen. Classical semantics proposes that our linguistic knowledge allows us to pick out a piece of information with a sentence, and our pragmatic competence tells us how that information should change our existing information. Dynamic semantics proposes that our linguistic knowledge directly encodes routines for changing information. Pragmatics still plays an enormous role in communication, since other changes can be inferred on the basis of how the sentence has changed the shared information. The uniform theory of conditionals proposed here employs a dynamic semantics, which is introduced in $\S 3.1$. The argument in favor of dynamic semantics here (§3.6) is interestingly different from those given elsewhere. Other arguments focus on how utterances of sentences change the conversationalists' shared information (e.g. Kamp 1981; Heim 1982; Groenendijk et al. 1996). The debate then centers on whether a classical conception of informational content and pragmatic information uptake can capture these changes (e.g. Stalnaker 1998; Lewis 2012; Dekker 2012). This can give the mistaken impression that the debate over dynamic semantics is simply a debate about the division of labor between semantics and pragmatics. The dynamic perspective on logical consequence and communication is important here, but the most important role played by dynamic meanings is in delivering an adequate compositional semantics. Without a dynamic semantics it is extremely difficult, if not impossible, to give a semantics to the if... then... structure and the unique morphology in subjunctive conditionals which captures the difference between indicatives and subjunctives, and produces an attractive logic.

Here is the route which connects these themes and a uniform theory of conditionals. My first goal will be to show that a uniform analysis which parallels the consistent extension/revision contrast is the best explanation of some key contrasts between the two kinds of conditionals (§2.1). After advancing a hypothesis about the linguistic encoding of this distinction (§2.2), I argue that the leading attempt at a uniform analysis (Stalnaker 1968, 1975) fails in several crucial ways $(\S 2.3)$. These difficulties are solved by the new analysis proposed

W. STARR

$03 / 23 / 13$ 
in $\S 3$. That analysis takes the dynamic semantics for indicative conditionals advanced in Gillies (2009) and Starr (2013a) and transforms it into a uniform theory of conditionals that explains the basic contrasts between the two varieties by paralleling the extension/revision contrast. On this theory, the antecedents of indicative and subjunctive conditionals propose different hypothetical changes to the information at stake in a conversation or inquiry. To explicitly encode this difference in the semantics of conditionals, it is necessary to construe the meanings of antecedents dynamically: in terms of their potential to change a body of information rather than in terms of their (contribution to) truth-conditions. The paper concludes by showing that the analysis of subjunctives offered here counts as a variably-strict one in the tradition of Stalnaker (1968) and Lewis (1973) and how it it remedies some serious obstacles for such an analysis. The paper also ends with a qualification of the idea that the kind of revision at work in subjunctive conditionals is likely not the same process by which we revise our beliefs after discovering they are wrong.

There are a few ideas from the philosophy of language that will be essential in what follows. As inquiry and conversation proceed, a body of information accumulates. Think of this information as what the agents are taking for granted in some way. A convenient model of this information can be stated in terms of a set of possible worlds (Stalnaker 1978: 86). Like information, this set distinguishes ways the world might be (possibilities in the set) from ways it isn't (possibilities excluded from the set). Call this set $c$, short for contextual possibilities or contextual information. Grice, Lewis, Stalnaker and others view this information as what is mutually taken for granted. ${ }^{8}$ This is how I will understand $c$. However, in $\S 3.2 \mathrm{I}$ will depart from the idea that $c$ is the only information that evolves as linguistic activities unfold.

\section{Stalnaker's Uniform Analysis}

One of the most basic contrasts between indicative and subjunctive conditionals is illustrated in (3) and (4).

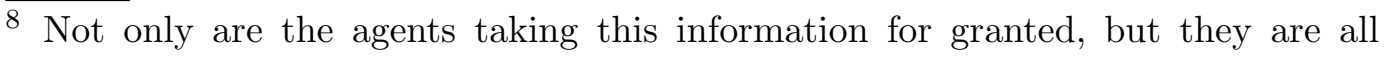
taking for granted that they are taking it for granted, and taking that for granted, etc. (Lewis 1969, 1979b; Stalnaker 1999, 2002; Clark 1996; Fagin et al. 1995: Ch.4).

W. STARR

$03 / 23 / 13$ 
(3) \# Bob never danced. If Bob danced, Leland danced.

(4) Bob never danced. If Bob had danced, Leland would have danced.

In both (3) and (4) the conditionals are interpreted against the information that precedes them. The indicative is anomalous when its antecedent is used to talk about a scenario incompatible with that information, unlike the subjunctive. This contrast suggests a hypothesis: indicative conditionals must be used to explore consistent extensions of a given body of information while subjunctive ones may be used to explore revisions of that body of information.

(3) and (4) are not the only data to be accounted for by a uniform theory of conditionals. The assertion of a subjunctive conditional often suggests something an ordinary indicative does not: ${ }^{9}$

The Subjunctive Suggestion Assertions of if Bob had danced, Leland would have danced often suggest that Bob didn't dance.

The reality of this suggestion is evidenced by the oddity of (5).

(5) \# Bob danced. If Bob had danced, Leland would have danced.

What is the source and status of this 'suggestion'? It is not an entailment and it is not a presupposition.

(6) If Jones had taken the arsenic, he would have just exactly those symptoms which he does in fact show (Anderson 1951:37)

(7) If the butler had done it, we would have found blood on the kitchen knife. The knife was clean; therefore, the butler did not do it. (Stalnaker 1975: 71)

In (6) the subjunctive conditional is used as an argument for the antecedent and so cannot be construed as presupposing or entailing the antecedent's falsity. (7) is a premise in a non-circular argument for the falsity of its antecedent and so cannot presuppose it. Since the second premise is ineliminable the subjunctive cannot alone entail the falsity of the antecedent. These examples make clear that there is no general 'suggestion of falsity' for subjunctive conditionals.

$\overline{9}$ I'll be a monkey's uncle if Bob dances more than suggests that Bob won't dance.

W. StarR

$03 / 23 / 13$ 
So, however this suggestion is tied to the conventional meaning of subjunctives, it needs to be weaker than a suggestion of flat out falsity.

\subsection{Stalnaker's Distinction}

Stalnaker's (1975: §3) way of drawing the indicative/subjunctive distinction sheds light on the phenomena described above.

Stalnaker's Distinction An indicative conditional focuses solely on antecedentworlds among the contextually live possibilities $c$, which represent what's being taken for granted in the discourse. A subjunctive conditional focuses on antecedent-worlds that need not be among those possibilities, that is they may be counterfactual from the perspective of the discourse.

(Stalnaker 1975: §3)

As Stalnaker (1975: 69) puts it "the idea [for indicative conditionals] is that when a speaker says if $A$, then everything he is presupposing to hold in the actual situation is presupposed to hold in the hypothetical situation in which $A$ is true." Subjunctive antecedents, on the other hand, signal that in saying if $A$ the speaker may be suspending some presuppositions and reaching beyond the contextually live possibilities (Stalnaker 1975: 70).

Stalnaker does not discuss the Subjunctive Suggestion, but the following explanation suggests itself. The choice between using a subjunctive and an indicative is the choice between a form that allows antecedent worlds from outside the contextual possibilities and a form which does not. Why would the speaker select the subjunctive instead of the indicative form? Because they wanted to allow antecedent worlds from outside $c$. Why would they want to do that? Either they think some antecedent worlds are outside $c$ or they shouldn't presuppose that the speaker thinks that all of the antecedent worlds are in $c$. The former kind of explanation correlates with contexts in which the Subjunctive Suggestion arises, while the latter correlates with contexts where it doesn't but a kind of neutrality about the possibility of the antecedent is desired, e.g. (6). This kind of abductive interpretation is familiar from other work in pragmatics (Hobbs et al. 1993), and explains why the Subjunctive Suggestion is both defeasible and a default. It is often the strongest assumption consistent with the context, but that can change either after more information comes in or when other information makes a weaker explanation more plausible. While

W. STARR

$03 / 23 / 13$ 
further work is necessary to properly formulate this proposal, its basic shape and promise is clear enough for present purposes. The focus here will be its semantic assumption: indicatives require antecedents within $c$, subjunctives do not. While I think this a plausible interpretation of Stalnaker, I will be considering the view on its explanatory merits alone. ${ }^{10}$

Consider how Stalnaker's Distinction bears on (3) and (4).

(3) \# Bob never danced. But, if Bob danced, Leland danced.

(4) Bob never danced. But, if Bob had danced, Leland would have danced.

In (3) the indicative conditional is being interpreted against presuppositions which are incompatible with Bob's having danced. But in uttering an indicative conditional a speaker is talking about a situation where those presuppositions hold and Bob danced. Since there is no such situation, this will yield a defective utterance - just as it is defective to utter My sister is running when it is common ground that I don't have a sister. By contrast, in (4) a speaker is talking about a situation where the presuppositions may be suspended, so there may indeed be a situation satisfying the constraints communicated by the speaker's utterance.

Lastly, Stalnaker's Distinction clearly connects the two species to two different ways of combining information. Indicatives involve combining two consistent bodies of information (the antecedent and $c$ ), while subjunctives involve combining two bodies of information that may very well be inconsistent (the antecedent and $c$ ).

${ }^{10}$ von Fintel $(1999 b)$ proposes a similar view where subjunctives presuppose that the space of worlds relevant for evaluating the antecedent are not a subset of $c$. As he notes, none of his data distinguishes this from the Stalnakerian view. Leahy (2011) proposes that indicatives presuppose the epistemic possibility of their antecedent, that subjunctives have no presuppositions and derives the Subjunctive Suggestion as an 'antipresupposition' using an epistemic logic. There seem to be counterexamples to this claim about indicatives: "I know that Bob danced. But I'm happy to humor you and consider the possibility that he didn't. If he didn't dance, Leland didn't dance either." Since the Leahy (2011) approach truly requires this assumption, I'm inclined to the abductive approach sketched above.

W. STARR

$03 / 23 / 13$ 


\subsection{The Ingredients of a Subjunctive Antecedent}

How is Stalnaker's Distinction linguistically encoded? To begin answering this question, I will attend to one prominent feature of subjunctive antecedents: they are marked with past tense morphology. ${ }^{11}$

(8) a. If Bob had danced, Leland would have danced

b. If Bob were to dance, Leland would dance

c. If Bob danced, Leland would dance

Surprisingly, this morphology does not have a genuinely past tense meaning. Out of context, the default reading of (8a) may very well concern a possibly counterfactual past event of Bob dancing. But, the antecedent may be coherently supplemented with tomorrow to yield an antecedent that concerns a possibly counterfactual future event.

(9) Bob died yesterday. If he had died tomorrow instead, he would have been 98 years old.

As evidenced by (11), this is not possible for a genuinely past tense reading of Bob had died, like that in (10).

(10) Yesterday I went to the Black Lodge. By the time I got there, Bob had died, but Cooper hadn't.

(11) I will go to the Black Lodge tomorrow. \# By the time I get there, Bob had died, but Cooper hadn't.

Thus, the past tense marking in subjunctive antecedents does not have a genuine past tense interpretation. ${ }^{12}$ This phenomenon is relevant to contrasting

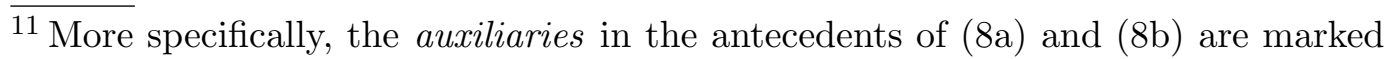
with what looks like past tense, and the same goes for the main verb in the antecedent of (8c). When multiple tenses are layered as in (8a) and (8b), the tense on the auxiliary determines the overall tense of the sentence. The acceptability of perfective sentences like By tomorrow, Bob will have danced makes this clear. Otherwise, such a sentence would be as unacceptable as \#Bob danced tomorrow.

${ }^{12}$ Parallel examples for (8a) and (8b) are available (Iatridou 2000). With subjunctives like $(8 \mathrm{~b})$ there is the additional difference that even with singular subjects the plural conjugation is used. In isolation $B o b$ were to dance is ungrammatical, though

W. STARR

$03 / 23 / 13$

10 
indicative and subjunctive conditionals since it does not occur in the former.

\# If Bob danced tomorrow, Leland danced tomorrow

At this point, three observations have accumulated. First, Stalnaker's Distinction: an indicative is concerned solely with antecedent-worlds among the contextually live possibilities while a subjunctive concerns possibly counterfactual antecedent-worlds. Second, the past tense morphology in the antecedents of subjunctive conditionals does not have its usual past tense meaning. Third, the past tense marking of indicative conditionals does have its usual past tense meaning. The contrast highlighted by the second and third observations offers a possible explanation of how Stalnaker's Distinction is linguistically encoded:

Counterfactuality Hypothesis The seemingly past tense morphology in the antecedents of subjunctive conditionals serves a modal function rather than a temporal one. It locates the antecedent event among a set of possibilities that may contain counterfactual ones, i.e. ones that may not be among the contextually live possibilities. Past tense morphology serves a purely temporal function in indicative antecedents, and so indicative antecedents remain concerned with the live contextual possibilities.

(Isard 1974; Lyons 1977; Iatridou 2000)

The basic idea is that these three observations are nicely explained by assuming a modal interpretation of past tense morphology in the antecedents of subjunctives gives rise to Stalnaker's Distinction. Any account of how subjunctive conditionals are put together must account for the fact that past tense morphology does not receive its normal temporal interpretation in subjunctive conditionals, but does in the antecedents of indicatives. Stalnaker's Distinction delineates a modal difference between these two kinds of antecedents. Both points are unified by the hypothesis that this past tense morphology takes on a modal interpretation. More specifically, instead of locating the antecedent event, e.g. Bob's dancing, in the past, it locates that event in a possibly counterfactual situation. There are a number of ways one might explain how past tense morphology alternates between these two interpretations. Though this is an important part of this semantics, it is not my focus here. The goal here is to formulate the analysis generally enough to be compatible with numerous ac-

The men were (supposed) to dance is acceptable. The latter sentence exhibits the same 'fake past' pattern in that it allows tomorrow, yesterday and today.

W. STARR

$03 / 23 / 13$ 
counts of these linguistic details. Indeed, my analysis will be general enough to cohere with a more nuanced approach proposed by Schulz (2007: §6.4). Schulz proposes that in simple past subjunctives, past tense morphology can be interpreted modally (what she calls subjunctive mood). However, in past perfect subjunctives past tense can be semantically vacuous, in which case have contributes a slightly different modal meaning (what she calls counterfactual mood). Schulz motivates this hypothesis with a wider set of data than considered here and formulates a fully compositional analysis based on it. There is much to recommend this analysis, but its differences with the Iatridou-style analysis are not relevant here. Accordingly, I will introduce a modal operator $\triangleleft$ called counterfactual expansion to model the modal contribution characteristic to subjunctive antecedents, leaving open the possibility of refining this operator further to parallel Schulz's subjunctive (Schulz 2007: 236) and counterfactual (Schulz 2007:240) moods. This simplification, as well as the simplified semantics of $\triangleleft$, will facilitate easier comparison with existing approaches. On my approach, a simple subjunctive conditional will be represented as (if $\triangleleft p$ ) $q$ and the corresponding indicative as (if $p$ ) $q$. The question is how the semantics of (if ·) · and $\triangleleft$ can be given to yield a uniform analysis of conditionals. I will begin by evaluating whether or not Stalnaker's proposal can provide a satisfactory answer to these questions. After arguing that it cannot, I will propose a semantics for $\triangleleft$ and combine it with the semantics for indicatives offered in Starr (2013a) and Gillies (2009) to yield a uniform analysis of conditionals. For simplicity, $\triangleleft$ will be modeled as a specialized sentential operator. Though, it is admittedly representing the contribution of something that is not really a sentential operator. Neither have or past tense take scope over fully-formed sentences, nor sentences of arbitrary complexity. Respecting this will require some seemingly arbitrary stipulations about the syntax of $\triangleleft$ (see Appendix). A more detailed model with a syntactically richer representation language and a deeper investigation into the scopal properties of $\triangleleft$ is needed. Consider this, then, a first-approximation.

Before moving on it is worth acknowledging a tradition of analysis which attempts to give a standard past tense meaning to subjunctive antecedents after all (Skyrms 1974; Adams 1975a; Dudman 1981, 1983, 1984, 1988; Ippolito 2003; Arregui 2007, 2009). The basic idea is that the past tense in subjunctive antecedents scopes over the modal meaning: when I say if Bob had danced tomorrow I'm talking about what used to be possible, namely a future in which Bob danced. On this view, a subjunctive is a report about a past possibility.

W. STARR

$03 / 23 / 13$

12 
As von Fintel (2012: $§ 7)$ notes, this view seems prima facie unable to account for examples like If you had bet heads, you would have won. This conditional seems true when a coin has been tossed and ha come up heads. Yet, at no point prior were the (closest) worlds where you bet heads necessarily ones where you won (see Adams 1975a: Ch.4 for related objections). One should also worry about how the theory would apply to a language like Kalaallisut that distinguishes the two varieties with a morpheme lacking temporal content (Bittner 2011:35). ${ }^{13}$ Since Schulz (2007: §6.4) is able to capture all of the data used to motivate this real-past view, there is little that can be said quickly about the choice between them (though see Schulz 2007: §6.2.2). I do, however, wish to comment on Ippolito's (2003: 149-50) claim that an Iatridoustyle analysis is incapable of capturing examples like (9). The concern is that one of the two past tense morphemes will still have a past tense meaning, which should conflict with tomorrow. However, it is open to Iatridou to hold that both past tense morphemes receive a modal interpretation and that $\triangleleft \triangleleft \mathrm{p}$ is equivalent to $\triangleleft p$. A Schulz-style analysis also captures these examples. With this issue addressed and some motivations for a modal analysis of subjunctive antecedents aired, I turn to the semantics of modal antecedents.

\subsection{A First Attempt at Uniformity: Stalnaker (1975)}

Stalnaker $(1968,1975)$ provided one of the earliest and most successful attempts at a uniform analysis of conditionals. ${ }^{14}$ This analysis treats (if · ) in terms of a two-place sentential connective $>$, whose semantics is given in (13).

\section{Stalnakerian Semantics for Both Species}

(13) a. $\phi>\psi$ is true at $w$ if and only if $\psi$ is true at all of the $\phi$-worlds most similar to $w$

b. $\llbracket \phi>\psi \rrbracket_{c, f}=\left\{w \mid f\left(w, \llbracket \phi \rrbracket_{c, f}\right) \subseteq \llbracket \psi \rrbracket_{c, f}\right\}$

The set selection function $f$ takes a world $w$ and a proposition $p$ and delivers

$\overline{13}$ See also Van linden \& Verstraete (2008: $\$ 4)$ who suggest that even tensed languages commonly use non-temporal morphology to mark subjunctive antecedents. Murray (p.c.) also informs me that Cheyenne uses identical tense/aspect morphology in indicative and subjunctive antecedents, but a distinct hypothetical mood for the two.

${ }^{14}$ See Nolan (2003) and Williams (2008) for related approaches.

W. STARR

$03 / 23 / 13$ 
the set of $p$-worlds most similar to $w .{ }^{15}$ This selection must obey some basic constraints (see Appendix) but must be further determined by context. Stalnaker's core idea is that the assertion of an indicative conditional is interpreted with a further selection constraint. Given a set $c$ of contextual possibilities, he requires that "if the conditional is being evaluated at a world in $[c]$, then the world[s] selected must, if possible, be within $[c]$ as well" (Stalnaker 1975:69). The idea is that in evaluating an indicative conditional at a world compatible with what's being taken for granted (represented by $c$ ), the selection of antecedent worlds should preserve what's taken for granted. Stalnaker (1975) does not specify exactly which proposition results from this constraint, but says merely that if $w \in c$ then $f\left(w, \llbracket \phi \rrbracket_{c, f}\right) \subseteq c$ (Stalnaker 1975: 69). How can this remark be used to provide such a specification? When given a proposition $p, f$ selects $p$-worlds. The formal theory captures this by requiring that $f(w, p) \subseteq p$. Stalnaker's constraint can therefore be met by requiring that if $w \in c$, the conditional is evaluated not by considering $f\left(w, \llbracket \phi \rrbracket_{c, f}\right)$ but rather by considering $f\left(w, \llbracket \phi \rrbracket_{c, f} \cap c\right)$. But this does not yet fully specify a proposition. What happens with worlds not in $c$ ? It is natural to assume that in this case the constraint does not apply, so $f$ operates as in (13b). Stalnaker's proposal then seems to be that while the semantic content of an indicative conditional is given by (13), the assertion of an indicative in a context will express (14).

\section{Stalnakerian Pragmatics for Indicatives}

a. $\phi>\psi$ is true in $c$ at $w$ iff:

(i) If $w \in c, \psi$ is true at all of the $\phi$-worlds in $c$ most similar to $w$

(ii) If $w \notin c, \psi$ is simply true at all of the $\phi$-worlds which are most similar to $w$

$$
\begin{aligned}
& \text { b. } \quad \llbracket \phi>\psi \rrbracket_{c, f}=\left\{w \mid f\left(w, \llbracket \phi \rrbracket_{c, f} \cap c\right) \subseteq \llbracket \psi \rrbracket_{c, f} \text { if } w \in c \&\right. \\
& \left.f\left(w, \llbracket \phi \rrbracket_{c, f}\right) \subseteq \llbracket \psi \rrbracket_{c, f} \text { if } w \notin c\right\}
\end{aligned}
$$

Unfortunately, (14) does not embody Stalnaker's Distinction. Recall that ac-

${ }^{15}$ Stalnaker makes the uniqueness assumption: for all $w$ and $p$ there is at most one $p$-world most similar to $w$. The above remains neutral on this matter by universally quantifying over a set of the most similar worlds, be it a singleton or more populous set. Throughout, I will translate Stalnaker's discussion of $f$ into a setting that does not assume uniqueness.

W. STARR

$03 / 23 / 13$ 
cording to Stalnaker's Distinction an indicative conditional makes a claim only about antecedent worlds within $c$. (14) is importantly different. It mentions two kinds of antecedent worlds, those within $c-f(w, \llbracket \phi \rrbracket \cap c)$ - and those that may be outside of $c-f(w, \llbracket \phi \rrbracket)$. So on this theory, indicative conditionals $d o$ not just concern antecedent worlds within $c$. But this distinction was supposed to explain the two contrasts between indicatives and subjunctives discussed at the beginning of §2. Perhaps Stalnaker didn’t intend (14), but rather (15), as Nolan (2003:218) and Williams (2008:212) propose.

(15) a. $\phi>\psi$ is true at $w$ iff $\psi$ is true at all of the contextually live $\phi$-worlds most similar to $w$

b. $\llbracket \phi>\psi \rrbracket_{c, f}=\left\{w \mid f\left(w, \llbracket \phi \rrbracket_{c, f} \cap c\right) \subseteq \llbracket \psi \rrbracket_{c, f}\right\}$

On this version, the constraint applies both to worlds in $c$ and worlds outside of $c$. It is unlikely that Stalnaker, who stated his constraint conditionally meant it to apply unconditionally. And for good reason. The conditional proposition in (15) does not obey modus ponens. Consider a $w \notin c$ where $\phi \wedge \neg \psi$ is true, and further that $\psi$ is true at all of the $\phi$-worlds in $c$ that are most similar to $w$ (i.e. $\left.f\left(w, \llbracket \phi \rrbracket_{c, f} \cap c\right) \subseteq \llbracket \psi \rrbracket_{c, f}\right)$. Then the assertion of an indicative $\phi>\psi$ will express a proposition that is true in $w$, yet $w$ is a world where $\phi$ is true and $\psi$ is false! ${ }^{16}$ So Stalnaker's analysis either can't explain the contrasts that are the subject of my investigation or it leads to incorrect truth-conditions.

There is a proposition that could serve as the content of an indicative conditional assertion that obeys modus ponens and Stalnaker's Distinction:

$$
\llbracket \phi>\psi \rrbracket_{c, f}=\left\{w \in c \mid f\left(w, \llbracket \phi \rrbracket_{c, f}\right) \subseteq \llbracket \psi \rrbracket_{c, f}\right\}
$$

Only antecedent-worlds from within $c$ are of concern to this proposition and it is false outside $c$. This saves modus ponens, but has worrisome consequences. First, (16) cannot be derived from a restriction on the selection function. The

$\overline{16}$ Nolan (2003: 222-3) suggests requiring $c$ to be knowledge helps, but at least within Stalnaker's view where the context set is a set of worlds rather than an accessibility relation, this does not help. All this guarantees is that the actual world won't provide the counterexample to modus ponens, which is no consolation. Further this conversationally unrealistic requirement would vitiate nearly all of the explanatory applications of Stalnaker's framework. Lycan (2001) chooses to give up and reject modus ponens. In the theory proposed below, neither concession is necessary.

W. STARR

$03 / 23 / 13$ 
constraint would have to apply generally and therefore make all assertions be about worlds in $c$. But then this approach would mistakenly apply the same constraint to subjunctive conditionals.

It is worth reminding oneself here that it is not plausible to say that indicatives and subjunctives express the same proposition, but differ just presuppositionally: uttering an indicative presupposes the contextual possibility of the antecedent. This option is foreclosed by the familiar point that conditionals of the two species can differ in truth value in the same context: if Oswald hadn't killed Kennedy, someone else would have vs. if Oswald didn't kill Kennedy, someone else did. Further, it gives up Stalnaker's Distinction and so the explanations which led us to seek a semantics that embodies that distinction.

The foregoing highlights problematic details in Stalnaker's analysis which have not been worked out. But I sympathize with the reservation that these problems of detail are not enough alone to reject the only uniform analysis we have. However, I will now connect this discussion with that of $\S 2.2$ to raise a more basic problem for Stalnaker's analysis. This problem, taken together with the new uniform analysis in $\S 3$ which is free of both problems, presents a strong challenge to the Stalnaker (1975) analysis.

Stalnaker (1975) gives a single semantic clause for both indicative and subjunctive conditionals, namely (13). However, he takes the assertion of an indicative conditional to be subject to a 'pragmatic constraint' on the selection function. This constraint entails that the assertion of an indicative conditional expresses an importantly different proposition, namely that listed in (14). Here is Stalnaker's clearest statement of the approach:

Both kinds of conditionals... have the same abstract semantics, but a contextdependent parameter of the interpretation - the selection function - is differently constrained by the different grammatical constructions. So, on this theory, the difference between the two kinds of conditionals is a semantic difference in two different senses, but a purely pragmatic difference in a third sense. The difference is semantic, first in the sense that there will normally be a difference in the proposition expressed by the contrasting conditional sentences, even when uttered in similar situations. And it is semantic also in the sense that the difference is marked by a conventional linguistic device (the tense/aspect/mood difference). But the distinction is pragmatic in that the device works by the way it constrains

W. STARR

$03 / 23 / 13$ 16 
features of the context. The semantic rule that gives the truth conditions of the conditional as a function of the contextual parameter will be the same for both kinds of conditionals (Stalnaker 2005: n.13).

The constraint is said to be pragmatic in the sense that it applies to features of the context. But it is hard to say why, in the modern sense, this is pragmatic (as opposed to Montague's 1970 sense). Pronouns require a salient referent. Past tense requires a salient past event. Indexical pronouns require a salient conversationalist. These facts about how words draw on context do not count as pragmatic in the Gricean sense. They do not follow from basic truths about the rational and cooperative nature of the conversationalists. These are facts about the constant function of particular morpheme. As such, they belong to compositional semantics. ${ }^{17}$ Why, then, did Stalnaker (1975) not propose a meaning for the subjunctive mood and construe the different propositions expressed by subjunctive and indicative conditionals as a function of this mood's contribution? Once it is recalled what the mood's contribution was, it becomes clear that the difference between subjunctive and indicative propositions cannot be derived from this contribution and Stalnaker's semantics for the if... then... structure. As I discussed in $\S 2.2$, subjunctive mood seems to be encoded using the apparent past tense, or the perfect have, in the antecedent of subjunctives. Its job is to allow subjunctives to access worlds outside $c$. Yet, Stalnaker's semantic clause for if... then... already allows antecedent worlds outside $c$ ! He tries to limit indicatives to antecedent-worlds in $c$ by placing a special constraint on them. But, from the perspective of morphology and compositional semantics, this is backwards. The semantic clause for if... then... should be limited to antecedent worlds in $c$ unless the antecedent contains a morpheme that allows it to expand beyond $c$. It is only a theory of this form that can correctly correlate the linguistic differences with the semantic differences between the two kinds of conditionals. Just such a theory will be sketched in the following section.

$\overline{17}$ Stalnaker's 'pragmatic' constraint would have to apply sub-sententially: If Bob is dancing, then Laura would be dancing if she were here. 


\section{A New Uniform Analysis}

Stalnaker's approach to conditionals takes the meaning of a sentence to be its truth-conditions. In sketching an alternative, I will begin by describing the more general perspective on meaning that has come to be known as $d y$ namic semantics. After stating a semantics for all if...then... structures in this framework, I will provide a semantics for the $\triangleleft$ operator proposed in $\S 2.2$. This will allow me to explain the contrasts between indicative and subjunctive conditionals, as well as the subjunctive suggestion, discussed in $\S 2$.

\subsection{Dynamic Semantics}

From a dynamic perspective, the meaning of a sentence is the characteristic role it plays in linguistic activities (conversation, calculation, inquiry, thought, etc.). On the Stalnakerian model of these activities, their progress is captured by an evolving body of mutual information, $c$. So the characteristic role a sentence plays can be stated as a function from one body of information to another. This takes the form of an equation $c[\phi]=c^{\prime}$, where the difference between $c$ and $c^{\prime}$ is the characteristic effect utterances of $\phi$ have on any body of information $c{ }^{18}$ (Read $c[\phi]$ as ' $c$ updated with $\phi$ '.) To be sure, actual particular utterances of $\phi$ may have many other, sometimes eclipsing, effects. But this equation is supposed to capture the common contribution the linguistic item makes to those utterances. For a basic illustration of this framework and formulation of a conditional semantics, it is convenient to consider a simple propositional logic with the familiar syntax, starting with a set of atomic sentences $\mathcal{A} t=\left\{\mathrm{p}_{0}, \mathrm{p}_{1}, \ldots\right\}$, and forming conjunctions, disjunctions and negations from them. A possible world can be treated as a valuation, i.e. an assignment of one truth-value, either 1 (True) or 0 (False), to every atomic sentence. The meanings of sentences are specified in the format discussed above:

\section{Definition 1 (Update Semantics)}

$$
\begin{aligned}
c[\mathbf{p}] & =\{w \in c \mid w(\mathbf{p})=1\} \\
c[\phi \wedge \psi] & =(c[\phi])[\psi]
\end{aligned}
$$

$$
\begin{aligned}
c[\neg \phi] & =c-c[\phi] \\
c[\phi \vee \psi] & =c[\phi] \cup c[\psi]
\end{aligned}
$$

Atomic sentences eliminate any possibilities incompatible with their truth.

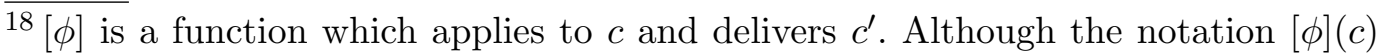
seems more appropriate, I will follow precedent. (Veltman 1996)

W. STARR

$03 / 23 / 13$ 
Negation eliminates all possibilities compatible with its scope. A conjunction updates $c$ with the first conjunct and then updates the resulting information with the second conjunct. Disjunction separately updates $c$ with each of its disjuncts and forms their union. To be sure, these clauses look different from the corresponding clauses of a non-dynamic possible worlds semantics. But it turns out those non-dynamic clauses are a consequence of Definition 1, provided one has a suitable definition of truth in the dynamic system. While many dynamic semanticists focus on another concept called support, truth can be seen as a special case of support (Starr 2010).

\section{Definition 2 (Support, Truth in $w$ )}

(1) Support $c \vDash \phi \Leftrightarrow c[\phi]=c \quad$ (2) Truth in $w w \vDash \phi \Leftrightarrow\{w\}[\phi]=\{w\}$

Sometimes the information provided by a sentence is already contained in $c$ (explicitly, or implicitly), in which case $c$ is said to support that sentence in the sense that an assertion of that sentence would be supported by $c .{ }^{19}$ Being true at a world just amounts to being supported by perfect information about that world. After all, if one were to have perfect information about $w$, and the information provided by $\phi$ was already contained in that information, $\phi$ must correspond to the facts in $w$. Since support is taken to be the key semantic concept, entailment is typically defined in terms of support, rather than truth.

Definition 3 (Entailment v1) $\phi_{1}, \ldots, \phi_{n} \vDash \psi \Leftrightarrow \forall c: c\left[\phi_{1}\right] \cdots\left[\phi_{n}\right] \vDash \psi$

The basic idea is that if incrementally adding $\phi_{1}, \ldots, \phi_{n}$ to any state of information results in a state that supports $\psi$, then $\phi_{1}, \ldots, \phi_{n}$ entail $\psi$. Interestingly, if this definition is restricted to states of perfect information (singleton sets of worlds), classical entailment results.

With truth now defined, propositions are also easily defined:

Definition 4 (Propositional Content) $\llbracket \phi \rrbracket=\{w \mid w \vDash \phi\}$

As previously mentioned, Definitions 1 and 4 yield as corollaries the clauses of

19 The present model does not distinguish implicit and explicit information, i.e. information agents have immediate access to versus information they could access by communication or thought. Models that make this distinction exist but introduce unnecessary complications here (Fagin et al. 1995: Ch.9).

W. STARR

$03 / 23 / 13$ 
standard possible worlds semantics:

Corollary 1 (Possible Worlds Semantics)

(3) $\llbracket \phi \wedge \psi \rrbracket=\llbracket \phi \rrbracket \cap \llbracket \psi \rrbracket$

$$
\begin{aligned}
\llbracket \neg \phi \rrbracket & =W-\llbracket \phi \rrbracket \\
\llbracket \phi \rrbracket \cup \llbracket \psi \rrbracket & =\llbracket \phi \rrbracket \cup \llbracket \psi \rrbracket
\end{aligned}
$$

However, the dynamic semantics is not yet more powerful or general. One could have started with the possible worlds semantics and derived the update semantics as a corollary by using the following as a definition of update:

\section{Stalnaker's Pragmatic Update Equation $c[\phi]=c \cap \llbracket \phi \rrbracket$}

Indeed, Stalnaker (1978: 86-7) proposed that this equation captures a necessary pragmatic consequence of successful assertions. One way to argue for a dynamic semantics is to find a sentence whose meaning does not seem to obey this equation. This disobedient behavior could involve a non-eliminative update, i.e. one where $c[\phi] \nsubseteq c$. Or, it could involve an update that does not amount to intersecting $c$ with $\llbracket \phi \rrbracket$, but rather some other proposition. ${ }^{20}$ Both kinds of argument can be (and usually are) challenged by offering an alternative pragmatic account of the non-eliminative update or by offering a more nuanced theory of the proposition expressed by $\phi$, perhaps one involving context-sensitivity. I shall provide a different kind of argument below. First, that argument will be about a sub-sentential context update and is therefore less plausibly rebutted with a pragmatic explanation. Second, that argument appeals to constraints of compositionality more than expressive limits of static meanings. This argument will be detailed in §3.6.

\subsection{Conditional Semantics}

Update semantics allows one to state meanings that do not take the familiar form of adding information to $c$. One example is a test, and my proposal

$\overline{20}$ This way of stating the dialectic uses the properties of eliminativity and continuity often discussed in the context of dynamic semantics (van Benthem 1986, 1991; Groenendijk et al. 1996; van Benthem et al. 2011). Rothschild \& Yalcin (2013) have shown that idempotence and commutativity provide a more general characterization of the updates that don't obey the equation above. This development leads to the same basic debate with different properties guiding it. It is therefore equally orthogonal to the kind of argument for dynamic semantics provided below.

W. STARR

$03 / 23 / 13$

20 
is that conditional meanings are of this kind. Instead of eliminating worlds meeting some condition, tests check whether the existing information has a particular feature. If it does not, failure results: a transition to the state of absurd information, $\varnothing$. Passing the test permits the existing information to proceed unchanged. A first approximation of the conditional semantics helps to illustrate this idea:

\section{Definition 5 (Conditional Semantics, v1)}

$c[($ if $\phi) \psi]= \begin{cases}c & \text { if } c[\phi] \vDash \psi \\ \varnothing & \text { otherwise }\end{cases}$

According to this definition, a conditional tests whether the background information $c$, together with the antecedent, supports the consequent. This is a close relative of the strict conditional theory first proposed by Lewis (1914): the consequent is a necessary consequence of the antecedent. However, there are three new twists. First, the necessity is restricted to the worlds in $c$. Second, the kind of consequence at stake is dynamic: the consequent provides no new information when one has the information $c[\phi]$. Third, by using a dynamic notion of consequence and attending to the presuppositions of indicative conditionals, this theory provides a logic of conditionals that is far more plausible than Lewis (1914). While Starr (2013a) argues at length that this logic has a reasonable claim to being the best available, I will summarize that argument below. This summary will motivate modified versions of Definitions 3 and 5 which reflect the presuppositional features of indicative conditionals.

The material conditional validates the following suspect forms of reasoning.

(17) Material Antecedent (MA) $\neg \phi \vDash \phi \supset \psi$

Bob didn't dance. So, if Bob danced, he was a turnip.

Material Negation (MN) $\neg(\phi \supset \psi) \vDash \phi$

It's not true that if God exists, he's a turnip. So, God exists.

Stalnaker $(1968,1975)$ and Adams $(1975 b)$ propose theories that invalidate (17), but at the cost of invalidating the plausible patterns of reasoning in (18):

(18) Import-Export $\phi_{1} \rightarrow\left(\phi_{2} \rightarrow \psi\right) \exists \vDash\left(\phi_{1} \wedge \phi_{2}\right) \rightarrow \psi$ Antecedent Strengthening $\phi_{1} \rightarrow \psi \vDash\left(\phi_{1} \wedge \phi_{2}\right) \rightarrow \psi$

W. STARR

$03 / 23 / 13$ 


\subsection{Conditional Semantics}

Disjunctive Antecedents $\left(\phi_{1} \vee \phi_{2}\right) \rightarrow \psi \vDash\left(\phi_{1} \rightarrow \psi\right) \wedge\left(\phi_{2} \rightarrow \psi\right)$

Transitivity $\phi_{1} \rightarrow \phi_{2}, \phi_{2} \rightarrow \psi \vDash \phi_{1} \rightarrow \psi$

Contraposition $\phi \rightarrow \psi \vDash \neg \psi \rightarrow \neg \phi$

Adams and Stalnaker attempt to justify this by offering counterexamples to the last four patterns in (18). ${ }^{21}$ However, upon further inspection, all of these attempted counterexamples involve the evaluation of an indicative conditional in a context where its presupposition is not met (Gillies 2009: §7, Starr 2013a). They involve the evaluation of an indicative conditional in a context where the antecedent is taken to be impossible. ${ }^{22}$ Yet, indicative conditionals clearly presuppose that their antecedents are possible:

(19) Sue never smoked. \# If she smoked, she didn't run.

This opens the door for an analysis that renders valid all the patterns in (18) and gives a principled explanation of the cases where those patterns breakdown. As long as it also has something to say about MA and MN, it seems quite clear that such an analysis would count as giving the best logic or natural language indicative conditionals. This kind of analysis requires two modifications to that given above: a semantics that reflects the presuppositions of indicative conditionals and a definition of consequence that does not count cases of presupposition failure against an inference pattern. Both modifications are easily articulated in update semantics where presuppositions can be treated as definedness conditions on updates (Heim 1982). For present purposes, this amounts to modifying the conditional semantics from Definition 5 as follows.

\section{Definition 6 (Conditional Semantics, v2)}

$c[($ if $\phi) \psi]= \begin{cases}c & \text { if } c[\phi] \neq \varnothing \& c[\phi] \vDash \psi \\ \varnothing & \text { if } c[\phi] \neq \varnothing \& c[\phi] \not \models \psi \\ \text { Undefined } & \text { otherwise }\end{cases}$

Strawsonian consequence (von Fintel 1999a; Starr 2013a) provides just the sort

${ }^{21}$ Adams (1975b: 11.8$)$ doubts Import-Export. But even his sympathizers are unmoved: McGee (1989) and Bennett (2003: $§ 40)$ who calls this pattern if-and.

${ }^{22}$ Veltman (1986) and Warmbrōd $(1981 b, 1983)$ make this observation but propose pragmatic views about the kind of infelicity that results.

W. STARR

$03 / 23 / 13$

22 
of consequence related needed here. On this understanding of consequence, one only considers contexts where an update with the premises followed by the conclusion are defined. This is a small modification of Definition 3:

Definition 7 (Entailment v2) $\phi_{1}, \ldots, \phi_{n} \vDash \psi \Leftrightarrow \forall c^{*}: c^{*}\left[\phi_{1}\right] \cdots\left[\phi_{n}\right] \vDash \psi$ - $c^{*}$ is any context s.t. $c\left[\phi_{1}\right] \cdots\left[\phi_{n}\right][\psi]$ is defined.

This analysis quite clearly invalidates MN, but it is not obvious that it makes the correct predictions about MA and other patterns, like $\psi \vDash($ if $\phi) \psi$ and $\psi \vDash \neg(($ if $\phi) \neg \psi)$. I think these issues can be quite compellingly addressed, but I leave that to Starr (2013a).

It might be thought that this approach to the logic of indicative conditionals is a bad fit for the project of this paper: a uniform theory of conditionals. First, the conditional semantics seems to encode something particular to indicative conditionals (their presupposition) into the basic conditional form. Second, it is a strict conditional theory, but I suggested in the introduction that one wants a variably-strict semantics for subjunctive conditionals. In $\S 3.4$ I will show that an appropriate semantics for $\triangleleft$ neutralizes this presupposition and yields a variably-strict semantics for subjunctives. Before that, I would like to conclude this section with a more detailed discussion of the proposal that conditionals express tests in an update semantics.

\subsection{Conditionals as Tests}

According to the semantics in Definitions 5 and 6, the conditional does not do its job in communication or inquiry by asserting the proposition that the antecedent plus the background information supports the consequent. Accepting the conditional and moving on in inquiry does signal that this condition holds, but in a different way. The fact that it cannot be seen as the assertion of a conditional proposition is clear when one considers what proposition a conditional does express on the proposed semantics (recall Definition 4). A world $w$ is in $\llbracket($ if $\phi) \psi \rrbracket$ if updating $\{w\}$ with that conditional returns $\{w\}$. On the present semantics that will occur when $\{w\}[\phi] \vDash \psi$. On both versions of the semantics, this happens if $w$ is a $\phi \wedge \psi$-world. On the version without presupposition, this also happens if $w$ is a $\neg \phi$-world. ${ }^{23}$ So on the version without presupposition, the proposition expressed by a conditional is the material

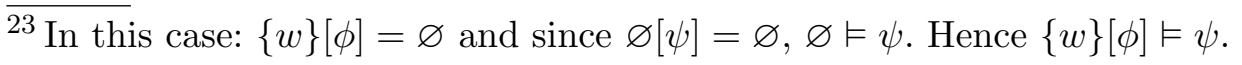

W. STARR

$03 / 23 / 13$ 
conditional: $\llbracket($ if $\phi) \psi \rrbracket=\llbracket \neg \phi \vee \psi \rrbracket$. On the version with presupposition, the proposition will be undefined when $w$ is a $\neg \phi$-world. So the best one gets is partial-truth conditions: (if $\phi$ ) $\psi$ is true when $\phi \wedge \psi$ is true, false when $\phi \wedge \neg \phi$ is true and undefined otherwise. ${ }^{24}$ However one works out the idea of a partial proposition, neither version obeys Stalnaker's Pragmatic Update Equation: $c[($ if $\phi) \psi] \neq c \cap \llbracket$ (if $\phi) \psi \rrbracket$. Suppose $c$ contains one $\phi \wedge \neg \psi$-world $w_{1}$ and one $\phi \wedge \psi$-world $w_{2}$. The assertion of (if $\left.\phi\right) \psi$ performs a test which fails for this $c$, and so $c[($ if $\phi) \psi]=\varnothing$, while $c \cap \llbracket$ (if $\phi) \psi \rrbracket=\left\{w_{2}\right\}$. It is worth noting, as Gillies (2009) does, that there is a context-sensitive assignment of propositions to sentences that satisfies this equation:

\section{Static Strict Conditional $\llbracket($ if $\phi) \psi \rrbracket_{c}=\left\{w \in c \mid c \cap \llbracket \phi \rrbracket_{c} \subseteq \llbracket \psi \rrbracket_{c \cap \llbracket \phi \rrbracket_{c}}\right\}$}

The proposition this conditional expresses is either $c$ or $\varnothing$, depending on whether or not the antecedent taken together with $c$ classically entails the consequent (when interpreted against that augmentation of $c$ ). Accordingly, a pragmatic update of this proposition by intersection with $c$ will behave exactly like the dynamic conditional: as a test. Strictly speaking, this preserves the idea of the conditional expressing a proposition, but it does not fit with the static idea that one makes assertions by expressing propositions and felicitous assertions must provide some information, i.e. eliminate at least one world from $c$ (see Stalnaker 1978: 88). I think this makes a static semantics a bad fit for the kind of strict-conditional semantics proposed here, but it also highlights a very natural question about any test semantics for conditionals. Don't successful utterances of them often provide information? How can a test semantics capture this? (20) seems to illustrate that a conditional can be used to eliminate $\phi \wedge \neg \psi$-worlds from $c$.

(20) (Context: I'm hosting a party and you've been mingling widely.)

$M e$ : I'm trying to find guests without drinks. Is Ulysses here?

You: I don't know. But he's quite a lush, so if he is here, he has a drink.

Me: \# That's an absurd thing to say! Nothing we've said so far rules out him being here without a drink!

$\overline{24}$ There is a long history of authors who have endorsed these truth-conditions but failed combine it with a plausible logic. Starr (2013a) argues that this is an additional interest of the present semantics.

W. STARR

$03 / 23 / 13$ 
It is my second utterance in (20) that seems absurd! Doesn't the test view predict that this absurd response should be reasonable? As stated, the test view is indeed incomplete, but it is still promising. Perhaps some uses of conditionals, like clarifications, target mutual information $c$. But it seems more common for them to target other bodies of information, like the speaker's own beliefs, common knowledge or information possessed by other salient individuals. So it seems that an update semantics should not limit its view to $c$. Nonetheless, these alternative bodies of information are still constrained by $c$. One cannot just target any bodies of information, but only those which are presupposed to exist. For example, it is presupposed that the speaker has some private beliefs $i_{s}$ but since it is not mutual information what all these beliefs are, $c$ and $i_{s}$ may differ. On this view, conditionals are interpreted from the perspective of $c$, but not always with respect to $c$. There is a set of bodies of information which $c$ makes available: $I_{c}=\left\{c, i_{0}, \ldots, i_{n}\right\} .{ }^{25}$ Here is the most conservative way of integrating this idea with the conditional semantics above. Read the $c$ in the semantics not as mutual information, but rather as some member $i$ (or members) of $I_{c}$, and leave it up to pragmatics to discern how testing these bodies of information could impact what is mutually taken for granted. It is not so hard to see how this pragmatic story would go. Assuming the speaker is being cooperative and rational, they will be speaking about an $i$ that is relevant and accessible to them. In this case, it is natural to allow the indicated properties of $i$ to be mirrored in $c$. By asserting the conditional in (20), you thereby proposed to have a particular property mirrored in the mutual information, namely that it lacks worlds where Ulysses is here without a drink. And thus tests can lead to an increase in the mutual information. Less conservative ways of integrating $I_{c}$ with the above semantics is to actually change the semantics. One can continue to outsource the link between an $i$ and $c$ to pragmatics, or explicitly require it:

\section{Conditionals in an Information Network (Two Options)}

a. $c[($ if $\phi) \psi]=\left\{w \in c \mid \exists i \in I_{c}: i[\phi] \vDash \psi\right\}$

b. $c[($ if $\phi) \psi]=\left\{w \in c \mid \exists i \in I_{c}: i[\phi] \vDash \psi \& c[\phi] \vDash \psi\right\}$

On the latter semantics, even though the test imposed by (if $\phi) \psi$ would fail, it is clear that the absurd state is not the intended result. Instead, the intended result was the minimal modification of $c$ needed to make it have the indicated

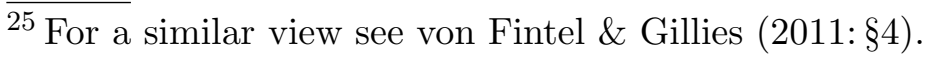

W. STARR

$03 / 23 / 13$ 
feature of $i$. In (20), this is the elimination of worlds where Ulysses is at the party but not drinking. I will not take a stand on which of these approaches is best, though it is discussed more in Starr (2013a).

It might be objected that this is an awfully complicated way of communicating. But the complications add a flexibility and power which we know natural language has, and more traditional, proposition-based views lack. The speaker's utterance can achieve its intended effect without specifying exactly which body of information lacks $\phi \wedge \neg \psi$-worlds. On a traditional view where the conditional expresses a proposition about some body of information, one cannot know what was communicated by the conditional until one knows which body of information it was about. Furthermore, the effect of aligning $c$ and $i$ would not happen just by the acceptance of the proposition that $i$ lacked $\phi \wedge \neg \psi$ worlds. Pragmatic reasoning parallel to that which supports the test semantics would be needed anyway.

\subsection{The Semantics of Counterfactual Expansion $\triangleleft$}

My aim is to formulate a semantics of conditionals that obeys Stalnaker's Distinction, which says that the antecedent of a subjunctive conditional concerns worlds that may not be among $c$, while an indicative conditional concerns worlds that are among $c$. The indicative conditional semantics from $\S 3.2$ has the later feature: only $\phi$-worlds in $c$ are considered in evaluating (if $\phi) \psi . \S 2.2$ claimed that the antecedent of a subjunctive a modal element $\triangleleft$. So the next step is to formulate a semantics for $\triangleleft$ that allows (if $\triangleleft \alpha$ ) $\psi$ to consider $\alpha$-worlds that may not be among $c$. My proposal is this:

What $\triangleleft \alpha$ Does Given $c, \triangleleft \alpha$ returns a set of $\alpha$-worlds $c^{\prime}$.

- If $w \in c$ is an $\alpha$-world, then $w \in c^{\prime}$.

- If $w$ isn't an $\alpha$-world, one or more alternative $\alpha$-worlds are placed in $c^{\prime}$. These alternatives may or may not be selected from $c$.

Previous semantic analyses of subjunctive conditionals provide different methods for selecting alternative $\alpha$-worlds. For example, the popular similarity analysis developed by Stalnaker (1968) and Lewis (1973) suggests that they are the $\alpha$-worlds most similar to $w \cdot{ }^{26}$ Premise semantics, developed by Veltman

$\overline{26}$ This formulation makes the limit assumption which Lewis (1973: $§ 1.4)$ officially rejected: there is a set of $\alpha$-worlds most similar to $w$. Though some reasons for adopt-

W. STARR

$03 / 23 / 13$

26 
(1985, 2005) and Kratzer (1989), and Pearl's (1996) structural equation semantics provide a different account that requires looking 'inside' $w$ and seeing how reversing $\alpha$ 's falsity would produce a different world or worlds. While my preference is for something like the later approaches (Starr 2013b), the dividing issues do not directly impact the project of defining a $\triangleleft$ operator with the behavior sketched above. The more familiar Lewis-Stalnaker analysis will suffice. The simplest and most direct formulation of the similarity analysis uses a selection function $f$, which takes a world $w$ and a proposition $p$, and returns the set of $p$-worlds most similar to $w$. Selection functions are required to meet some basic constraints. Stalnaker required (a)-(d) and Lewis (a)-(c). ${ }^{27}$

Definition 8 (Selection Functions) (Let $p, p^{\prime} \subseteq W$ and $w \in W$ )

(a) $\quad f(w, p) \subseteq p$

(b) $f(w, p)=\{w\}$, if $w \in p$

(c) $\quad f(w, p) \subseteq p^{\prime} \& f\left(w, p^{\prime}\right) \subseteq p \Longrightarrow f(w, p)=f\left(w, p^{\prime}\right)$

(d) $\quad f(w, p)$ contains at most one world success

strong centering

uniformity

uniqueness

Selection functions can be added to the framework of update semantics sketched above by taking sentences to operate on a pair consisting of a set of worlds $c$ and a selection function $f$, though for convenience I will write $c_{f}$ and talk as though this were the set $c$. The semantics for $\triangleleft$ can then be stated as follows:

\section{Definition 9 (Counterfactual Expansion $\triangleleft$ )}

Let $f$ be a selection function:

$c_{f}[\triangleleft \alpha]=\left\{w^{\prime} \mid \exists w \in c: w^{\prime} \in f(w, \llbracket \alpha \rrbracket)\right\}_{f}$

- $\triangleleft \alpha$ returns worlds which are among the $\alpha$-worlds closest to some $w \in c$

How does this semantics ensure the behavior described above? The first feature was that $\triangleleft \alpha$ keeps the $\alpha$-worlds from $c$. This is secured by the strong centering

ing it exist, it's a mere convenience here (Pollock 1976; Herzberger 1979; Warmbrōd 1982; Stalnaker 1984: 140-6).

${ }^{27}$ Again, I am putting aside the limit assumption. Stalnaker formulates the selection function as returning a single world rather than a singleton set. That implementation requires contortions (undefinedness or an impossible world) when evaluating $f(w, \varnothing)$, while the set selection formulation (Lewis 1973: §3.4) can just return $\varnothing$.

W. STARR

$03 / 23 / 13$ 
constraint. ${ }^{28}$ Consider any $\alpha$-world $w_{\alpha}$ from $c$. Strong centering requires that $w_{\alpha}$ be the closest $\alpha$-world: $f\left(w_{\alpha}, \llbracket \alpha \rrbracket\right)=\left\{w_{\alpha}\right\}$. So $w_{\alpha}$ is among the $\alpha$-worlds closest to some world in $c$, namely itself. The second feature of the analysis is that $\triangleleft \alpha$ replaces each $\neg \alpha$-world $w_{\neg \alpha}$ in $c$ with the $\alpha$-worlds closest to $w_{\neg \alpha}$, $f\left(w_{\neg \alpha}, \llbracket \alpha \rrbracket\right)$, and that these worlds may not be among $c$. Definitions 8 and 10 indeed leave open whether these worlds are in $c$. So these worlds may not be in $c$. Indeed, given the minimal constraints place on $f$, for any assertion of a subjunctive conditional, there will most likely be a compatible $f$ that selects some $\alpha$-worlds outside of $c$.

While I have just shown that $\triangleleft$ has the basic desired features, there is a feature of the analysis that deserves careful consideration. Recall that $\triangleleft$ corresponds to a modal interpretation of a past tense morpheme. For this reason, when we represent a subjunctive like (21) the antecedent should come out as $\triangleleft \mathrm{B} \wedge \triangleleft \mathrm{L}$. On the other hand, the antecedent of (22) is plausibly rendered as $\triangleleft(D \wedge S)$.

(21) If Bobby had danced and Leo had drank, Bobby would have danced

(22) If Bobby had danced and sung, he would have danced

(21) is so obviously true, that it is strange to assert. Indeed, it seems to instantiate a plausible logical validity:

Preservation $\vDash($ if $\phi \wedge \psi) \phi \wedge($ if $\phi \wedge \psi) \psi$

Yet, the current analysis allows the left conjunct to be false when its antecedent contains $\triangleleft$. Conjunction is sequential update, so (21)'s antecedent comes to: $\left(c_{f}[\triangleleft \mathrm{B}]\right)[\triangleleft \mathrm{L}]$. This involves first finding the B-worlds closest to worlds in $c$, and then finding the L-worlds closest to those B-worlds. The problem is that at this second step $f$ allows closest L-worlds which are not B-worlds. For any $\mathrm{B} \wedge \neg \mathrm{L}$-worlds that were in $c, f$ will find the closest L-worlds. But these may be outside of $c$, and so they may not be B-worlds. Thus, there will be $c_{f}$ 's for which $\left(c_{f}[\triangleleft \mathrm{B}]\right)[\triangleleft \mathrm{L}]$ contains $\neg \mathrm{B}$-worlds. But then there will be $c_{f}$ 's for which $\left(c_{f}[\triangleleft \mathrm{B}]\right)[\triangleleft \mathrm{L}] \not \models \mathrm{B}$. But then (if $\triangleleft \mathrm{B} \wedge \triangleleft \mathrm{L}$ ) $\mathrm{B}$ is not logically valid. The cause of this result should be obvious: instead of using $f$ once to find the closest $\mathrm{B} \wedge \mathrm{L}$ worlds, $f$ is being used once to find the closest B-worlds and then again to find the L-worlds closest to those B-worlds. This issue surfaces in another way in the Lewis-Stalnaker analysis. There, conjunctive antecedents are not problematic

${ }^{28}$ Weak centering would do as well: $w \in f(w, p)$ if $w \in p$.

W. STARR

$03 / 23 / 13$ 28 
because they are assimilated to $\triangleleft(A \wedge B)$. The problem instead arises with nested conditionals. The eminently plausible principle of Import-Export fails because in the nested conditional $\phi_{2}>\psi f$ is free to select $\neg \phi_{1}$-worlds. Yet, in the conjunctive antecedent, $f$ is required by Success to select $\phi_{1}$-worlds.

Import-Export $\quad$ if $\left.\phi_{1}\right)\left(\left(\right.\right.$ if $\left.\left.\phi_{2}\right) \psi\right) \exists \vDash\left(\right.$ if $\left.\phi_{1} \wedge \phi_{2}\right) \psi$

The present analysis validates Import-Export, but the issue re-emerges with Preservation. There are three possible responses to this issue I can imagine. The first is to defend the thesis that all conjunctive antecedents have the form $\triangleleft(\alpha \wedge \beta)$, but this is just to ignore the linguistic details that led to the postulation of $\triangleleft$ in the first place. The second option is to allow $\triangleleft \alpha$ to shift the assignment function to $f^{\llbracket \propto \rrbracket}$ which requires future uses in the antecedent to select $\alpha$-worlds. This option has much to recommend it, and is the approach taken in Starr (2010: Ch.3). However, I have since realized that it leads to a failure of modus ponens when subjunctives are nested in the consequent of a subjunctive, e.g. (if $\triangleleft A)(($ if $\triangleleft B) C$ ) and A won't entail (if $\triangleleft B) C$. The reason is that $\triangleleft \mathrm{A}$ would shift $f$, but $\mathrm{A}$ would not. It cannot, since otherwise you couldn't have non-vacuous counterfactuals: after accepting $\neg \mathrm{A}, \triangleleft \mathrm{A}$ would be forced to look for $\neg \mathrm{A} \wedge \triangleleft \mathrm{A}$-worlds! On the shifting approach if $\mathrm{A}$ is true in $w$, then (if $\triangleleft \mathrm{A})(($ if $\triangleleft \mathrm{B}) \mathrm{C}$ ) would require that all the B-worlds closest to $w$ according to $f^{\llbracket A \rrbracket}$ are $\mathrm{C}$-worlds. These closest worlds would all have to be $\mathrm{A} \wedge \mathrm{B}$-worlds. However, unembedded, (if $\triangleleft \mathrm{B}$ ) $\mathrm{C}$ will be evaluated with respect to $f$, which will require that the $\mathrm{B}$-worlds closest to $w$ according to $f$ are C-worlds. The difference is that these needn't all be $\mathrm{A} \wedge \mathrm{B}$-worlds. Indeed, $f$ can select a $\neg A \wedge B \wedge \neg C$-world. Since there are no genuine counterexamples to modus ponens, I regard this response as a dead-end. ${ }^{29}$ The third possibility is to deny Preservation. This position gains support from examples like this:

(23) Context: We see something off in the distance rummaging in a garbage bin. We agree it's a dog. I'm fond of pointing out how ecologically sound dogs indiscriminating dietary habits are.

29 The McGee (1985) 'counterexamples' to modus ponens are easily diagnosed and diffused on the dynamic semantics for conditionals described in $\S 3.2$ (Gillies 2004: 592-5). The similar subjunctive case from Briggs (2012: $§ 3.1)$ only arises in a structural equations framework, e.g. Pearl (2000: Ch.7). Since that is my preferred analysis of $f$, it is fortunate that my version of the structural equations semantics circumvents this problem by rendering the complex subjunctive false (Starr 2012).

W. STARR

$03 / 23 / 13$ 
a. If that dog were human, it would eat even more garbage.

b. [You take out your binoculars.] No way, look, it is a human!

c. Okay, well, if it were a dog and that dog were a human, then it would eat even more garbage.

Consider the consequent of $(23 \mathrm{c})$. It is clear that when we come around to evaluating this claim, the envisioned creature is not a dog, but rather a dogturned-human. After all, you would miss my point if you construed me as saying some dog would eat more garbage. Accordingly, it seems like this instance of Preservation is false in the context of (23):

(24) If it were a dog and that dog were human, then it would be a dog

This failure of Preservation has a specific feature: there is no world where both antecedents are true. In this case, it would seem that the second antecedent wins out. After all, it is at least clear that (25) sounds better than (24).

(25) If it were a dog and that dog were human, then it would be human

This is what the current semantics predicts:

Right Preservation $\vDash($ if $\triangleleft \alpha \wedge \triangleleft \beta) \beta$

No Left Preservation $\not \models$ (if $\triangleleft \alpha \wedge \triangleleft \beta$ ) $\alpha$

But there is still a story to be told about the cases where Left Preservation seems plausible. Those are cases where adopting $\beta$ doesn't force you to give up $\alpha$. Redefining $f$ in a way that builds on the following idea seems promising: when evaluating $\triangleleft \alpha$ you preserve any truths that don't depend on the falsity of $\alpha$. The structural equations approach I favor is an example of such an approach (Pearl 2000; Starr 2013b). In this setting, $\triangleleft \beta$ will only take you to $\neg \alpha$-worlds from an $\alpha$-world if $\alpha$ 's truth depended on $\beta$ being false, e.g. if $\alpha$ is 'Side $A$ of the teeter-totter is up' and $\beta$ is 'Side $B$ of the teeter-totter is up'. If $f$ had this feature, a restricted version of Left Preservation would hold:

Limited Left Preservation If $\exists w \vDash \alpha \wedge \beta$, then $\forall c_{f}: c_{f} \vDash($ if $\triangleleft \alpha \wedge \triangleleft \beta) \beta$

While I spell out the details of this approach to subjunctives in Starr (2013b), what I've said here should suffice to show that there is a workable alternative to either giving up modus ponens, misrepresenting subjunctive antecedents by

W. STARR

$03 / 23 / 13$ 
giving $\triangleleft$ wide scope or misrepresenting subjunctive antecedents by leaving out $\triangleleft$ altogether. I grant that the natural language examples bearing on Preservation are nuanced, but given how implausible the alternatives are, I believe this nuanced data adequately motivates the position I have just described.

\subsection{Unification}

The semantics for conditionals offered in $\S 3.2$ was originally proposed for indicative conditionals, but when combined with the semantics for $\triangleleft$ from $\S 3.4$ it yields a plausible semantics for subjunctive conditionals too. This is not immediately obvious, since indicative conditionals were assumed to presuppose that their antecedent was compatible with $c$ and this is not at all plausible for subjunctive conditionals. However, by plugging a subjunctive antecedent into that semantics, it becomes clear that this feature does not pose a problem.

$$
c_{f}[(\text { if } \triangleleft \alpha) \psi]= \begin{cases}c_{f} & \text { if } c_{f}[\triangleleft \alpha] \neq \varnothing_{f} \& c_{f}[\triangleleft \alpha] \vDash \psi \\ \varnothing & \text { if } c_{f}[\triangleleft \alpha] \neq \varnothing_{f} \& c_{f}[\triangleleft \alpha] \not \models \psi \\ \text { Undefined } & \text { otherwise }\end{cases}
$$

An indicative would presuppose that $c_{f}[\alpha] \neq \varnothing_{f}$, but a subjunctive has a different antecedent and so a different presupposition too: $c_{f}[\triangleleft \alpha] \neq \varnothing_{f}$. This presupposition can be met even when there are no $\alpha$-worlds in $c_{f}$. As long as $f$ can find an $\alpha$-world similar to one of the $\neg \alpha$-worlds in $c_{f}, c_{f}[\triangleleft \alpha] \neq \varnothing$.

\section{Subjunctive Visibility Presupposition}

- (if $\triangleleft \alpha) \psi$ presupposes that for some $w \in c_{f}, f(w, \llbracket \alpha \rrbracket) \neq \varnothing$.

- (if $\triangleleft \alpha) \psi$ presupposes that an $\alpha$-world is visible from $c_{f}$.

Conditionals with antecedents like $\triangleleft(\alpha \wedge \neg \alpha)$ will therefore be infelicitous, which seems at least as reasonable as rendering them vacuously true.

This uniform analysis embodies Stalnaker's Distinction (§2.1) perfectly. Indicative conditionals concern only the antecedent worlds within $c$, while subjunctives concern antecedent worlds that may lie outside of $c$. This is because it is always true that $c_{f}[\mathrm{~A}] \subseteq c$, but for certain $c_{f}$ 's, $c_{f}[\triangleleft \mathrm{A}] \nsubseteq c$. Embodying this distinction allows the present theory to explain the indicative and subjunctive contrasts discussed in $\S 2$ while validating modus ponens and tying 
these contrasts to the specific linguistic differences between the two varieties of conditionals. The present theory, unlike Stalnaker's Distinction alone, helps explain the oddity of (27).

(27) \# Bob always danced. If Bob had danced, Leland would have danced.

The first sentence limits the live possibilities to ones where Bob danced. The second sentence, according to Stalnaker's Distinction, suggests that some relevant Bob dancing-worlds may be beyond these live possibilities. But the current theory predicts that what the subjunctive actually asserts is at odds with this suggestion. After updating with the first sentence $c_{f}[\mathrm{~B}]$, only $\mathrm{B}$-worlds remain. However, in this context the antecedent of the subjunctive $\triangleleft \mathrm{B}$ will only concern those live B-worlds. Why? By Centering, $c_{f}[\mathrm{~B}][\triangleleft \mathrm{B}]=c_{f}[\mathrm{~B}]$. So selecting the subjunctive form in (27) suggests that some of the relevant Bworlds may be counterfactual (outside $c_{f}[\mathrm{~B}]$ ), and it goes on to say that none of them are counterfactual and that they are all L-worlds. While this is not a fully rigorous explanation, it illustrates that there is a clear tension on which a more detailed explanation could be based.

This uniform semantics amounts to an analysis which may have initially seemed impossible. It provides a strict analysis of indicative conditionals, but a variablystrict analysis of subjunctive conditionals. However, it does so with the same meaning for the if...then... structure. An indicative (if $\alpha$ ) $\psi$ says that $\neg \alpha \vee \psi$ holds throughout $c$. But a subjunctive (if $\triangleleft \alpha$ ) $\psi$ says that $\neg \alpha \vee \psi$ holds throughout $c[\triangleleft \alpha]$. In this sense, the set of worlds over which a subjunctive expresses a necessity varies depending on which antecedent is being considered. Hence, it is variably-strict. Logically, this difference impacts two inference patterns.

Antecedent Strengthening (if $\triangleleft \alpha) \psi \vDash$ (if $\triangleleft \alpha \wedge \triangleleft \beta) \psi$

Transitivity (if $\triangleleft \alpha_{1}$ ) $\alpha_{2}$, (if $\left.\triangleleft \alpha_{2}\right) \psi \vDash$ (if $\triangleleft \alpha_{1}$ ) $\psi$ 
Strict analyses validate both while variably-strict analyses validate neither. ${ }^{30}$ Antecedent Strengthening is indeed invalid on the present semantics. This pattern fails precisely because $\triangleleft \alpha_{2}$ may expand beyond the initially closest $\alpha_{1}$-worlds, and these may not be $\psi$-worlds. The failure of transitivity stems from the fact that it might be that (i) when you expand $c_{f}$ with $\alpha_{1}$ you only get $\alpha_{2}$-worlds and (ii) when you expand $c_{f}$ with $\alpha_{2}$ you only get $\psi$-worlds, but (iii) when you expand $c_{f}$ with $\alpha_{1}$ you get only $\psi$-worlds. Just consider an everyday light controlled by two binary switches. The light is on just in case the two switches are not in the same position. In the actual world, switch 1 is down, 2 is up and the light is on. In the minimally different world where switch 1 is up, 2 should remain up. The minimally different world where switch 2 is up is the actual world and the light is on. Yet, it does not follow that the minimally different world where switch 1 is up is a world where the light is on. Indeed, that is a world where both switches are up so the light is off. With this said, it is quite natural to interpret sequences of conditionals in a different way that leads to Transitivity sounding plausible. Rather than interpreting the second conditional with respect to $c_{f}$, it is natural to hear it as elaborating on the $\alpha_{2}$-worlds mentioned by the consequent of the first conditional. In this case, the second conditional is interpreted with respect to $c_{f}\left[\triangleleft \alpha_{1}\right]\left[\alpha_{2}\right]$. So, $\triangleleft \alpha_{2}$ will just (by Strong Centering) give us back that same set of worlds, so if all of them are $\psi$-worlds, it would have to follow that all of the worlds in $c_{f}\left[\triangleleft \alpha_{1}\right]$ are $\psi$ worlds. In that case, (if $\triangleleft \alpha_{1}$ ) $\psi$ will follow. Weak Transitivity is valid:

Weak Transitivity $\quad$ if $\left.\triangleleft \alpha_{1}\right) \alpha_{2}$, (if $\left.\triangleleft \alpha_{1}\right)\left(\left(\right.\right.$ if $\left.\left.\triangleleft \alpha_{2}\right) \psi\right) \vDash\left(\right.$ if $\left.\triangleleft \alpha_{1}\right) \psi$

This method of interpreting sequences of conditionals could be thought of as modal anaphora: one conditional sets up one set of possibilities and the subsequent one elaborates on it. While this is a prominent way of interpreting conditionals, it is not the way of connecting claims target by the dynamic no-

$\overline{30}$ Some strict accounts of subjunctive conditionals invalidate these patterns, but in a very specific case (e.g. Gillies 2007; von Fintel 2001). They rely on a case where the presuppositions of the premise are met but the conclusion's are not and cannot be accommodated. Thus, the truth of the premise does not guarantee the truth of the conclusion (and hence isn't dynamically valid either). However, the Strawsonian definition of entailment motivated in $\S 3.2$ does not count cases like this for or against an inference pattern. On this definition any strict analysis will validate Antecedent Strengthening and Transitivity. By contrast, on my semantics these patterns aren't even valid in the Strawsonian sense.

W. STARR

$03 / 23 / 13$ 
tion of logical consequence. Logical consequence is simply sequentially taking in the information semantically provided by each premise. While there is often more to understanding the structure of a discourse, one might think this additional structure is more appropriately handled in the pragmatic analysis of discourse. In $\S 4.2 \mathrm{I}$ will articulate one benefit of thinking this way and spell out formally how a pragmatic analysis of discourse structure could capture what is going on in Transitivity inferences that sound correct. There is a prominent, and positive, difference between the proposed logic of subjunctives and the Lewis-Stalnaker analysis. This concerns subjunctives with disjunctive antecedents, and will be discussed in §4.1.

Indicative and subjunctive conditionals can diverge in their truth-conditions. Since indicative conditionals are truth-valueless in worlds where their antecedents are false (§3.2), this had better be true on the proposed analysis. As mentioned earlier in this section, subjunctives still have a presupposition about the truth of their antecedent, but it is much weaker: (if $\triangleleft \alpha$ ) $\psi$ presupposes that for some $w \in c_{f}, f(w, \llbracket \alpha \rrbracket) \neq \varnothing$. This difference has an important consequence for the truth-conditions of subjunctives. While indicative conditional propositions are rarely defined (unless their antecedent is a tautology), subjunctive propositions are routinely defined, provided an $f$ is employed which can find an $\alpha$-world most similar to any given world. This follows from the definitions of truth and propositions from $\S 3.1$ and the semantics for subjunctive conditionals proposed above.

\section{Fact 1 (Simple Subjunctive Conditional Propositions)}

For non-conditional, non-modal $\psi$ :

(1) $\llbracket$ (if $\triangleleft \alpha) \psi \rrbracket_{f}$ is well-defined if $\forall w \in W, f(w, \llbracket \alpha \rrbracket) \neq \varnothing$

(2) When defined, $\llbracket($ if $\triangleleft \alpha) \psi \rrbracket_{f}=\left\{w \mid f(w, \llbracket \alpha \rrbracket) \subseteq \llbracket \psi \rrbracket_{f}\right\}$

The second part of this fact makes clear that on the present analysis subjunctive conditionals have the same truth-conditions as on the original LewisStalnaker account. This certainly makes clear that the truth-conditions of indicative and subjunctive conditionals are predicted to diverge on the present analysis, but it does not really capture the difference between (28a) and (28b) noted by Adams (1975b), and others following him.

(28) a. If Oswald didn't kill Kennedy, someone else did

b. If Oswald hadn't killed Kennedy, someone else would have

W. STARR

$03 / 23 / 13$ 
Adams says that (28a) is clearly true, but that, putting aside a massive conspiracy, (28b) is not. On the present analysis, (28a) is only true if, in the actual world Oswald didn't kill Kennedy and someone else did. But the subjunctive would also be true, since $f$ is assumed to obey Strong Centering. However, it is clear in the case Adams is describing, we are to evaluate the conditionals in a state of uncertainty. We know that Kennedy was murdered, and we think that Oswald probably killed Kennedy, but there's a slight chance he didn't. (28a) then says that in all of these worlds where Oswald didn't kill Kennedy, someone else did, since in all of those worlds Kennedy was murdered. This is captured on the present analysis not by talking about the truth of (28a) but about the fact that the context, as set up by our cultural knowledge, supports the indicative conditional. By contrast, (28b) requires something far stronger to be supported by the context. Like the indicative, it requires that someone else killed Kennedy in all of the live worlds where Oswald didn't. But it also requires that for each live world where Oswald did kill Kennedy, the most similar world where Oswald didn't commit this murder, someone else would have carried it out in his stead. Clearly any $f$ which does not observe the opinions of conspiracy theorists will lead to (28b) not being supported.

The final topic I wish to mention is the fact that the present analysis does not, so far, explicitly represent the contribution of the modals that appear in the consequent of subjunctive conditionals. As it turns out, this is harmless for would conditionals. Would is standardly analyzed to be a combination of a necessity modal woll with past tense (will being woll plus future tense). But the same data that led us to believe that the past tense in antecedents does not receive a genuinely past tense meaning also applies to the past tense component of would.

(29) If I were to run a marathon tomorrow, my sister would congratulate me afterwards

In (29) it is clear that the congratulations is not only in the future of the utterance time, but also in the future of the marathon running. Thus, it cannot be that the past tense component of would is contributing a real past tense meaning. So I propose to analyze would in terms of a combination of necessity and counterfactual expansion: would $A$ translates as $\square \triangleleft$ A. For $\square$, I have in mind the standard dynamic meaning for epistemic modals (Veltman 1996):

W. STARR

$03 / 23 / 13$ 


\section{Definition 10 (Necessity $\square$ and Possibility $\diamond$ )}

- $c_{f}[\square \phi]=\left\{w \in c \mid c_{f} \vDash \phi\right\}_{f}$

- $\square \phi$ tests that $c_{f}$ entails $\phi$

- $c_{f}[\diamond \phi]=\left\{w \in c \mid c_{f}[\phi] \neq \varnothing\right\}_{f}$

- $\diamond \phi$ tests that $c_{f}$ is consistent with $\phi$

Sticking $\triangleleft$ under this has no real effect, assuming Strong Centering. If $\square \mathrm{A}$ holds for $c_{f}$, then $\mathrm{A}$ is true in every world in $c_{f}$. But then $c_{f}[\triangleleft \mathrm{A}]=c_{f}$. So, the $\triangleleft$ doesn't really add anything. Furthermore, on the present semantics of conditionals, it can be proven that (if $\phi$ ) $\psi$ is equivalent to (if $\phi$ ) $\square \psi$. So it is harmless to represent a would-subjunctive as (if $\triangleleft A$ ) $B$ rather than (if $\triangleleft \mathrm{A}) \square \triangleleft \mathrm{B}$. It does, however, make a difference for should, could and mightsubjunctives. Adding $\triangleleft$ under $\diamond$ is not redundant. An update with $\diamond \mathrm{B}$ will fail when there are no live B-worlds in $c_{f}$. However, updating with $\diamond \triangleleft \mathrm{B}$ will not. This instead tests that there is at least one world in $c_{f}[\triangleleft \mathrm{B}]$. As long as $f$ can find some B-world similar to one of the live $\neg$ B-worlds, this test will be passed. So, the antecedent of (if $\triangleleft A$ ) $\diamond \triangleleft B$ will temporarily shift the context to the $\mathrm{A}$-worlds most similar to some live possibility. The consequent will then test that there is at least one world among these which is either a B-world, or that some world $f$-similar to it is a B-world. In this last step it becomes clear that (if $\triangleleft \mathrm{A}) \diamond \triangleleft \mathrm{B}$ does not require a $\mathrm{B}$-world in $c_{f}[\triangleleft \mathrm{A}]$ : it can expand to find one when necessary. By contrast $\neg($ if $\triangleleft A) \square \triangleleft \neg B$ requires that there are some B-worlds in $c_{f}[\triangleleft \mathrm{A}]$. So (if $\left.\triangleleft \mathrm{A}\right) \diamond \triangleleft \mathrm{B}$ does not entail $\neg$ (if $\left.\triangleleft \mathrm{A}\right) \square \triangleleft \neg \mathrm{B}$. However, the opposite does hold since if there are some B-worlds in $c_{f}[\triangleleft \mathrm{A}]$, then by Strong Centering $c[\triangleleft \mathrm{A}][\triangleleft \mathrm{B}]$ will contain at least those worlds and hence be non-empty. So unlike Lewis (1973), the present analysis does not validate Duality, yet unlike Stalnaker (1984: Ch.7), we are not forced to analyze might and could-subjunctives as involving a wide-scope epistemic modal. While the present approach improves on both by giving a genuinely compositional analysis of subjunctives, I regard it as still tentative. There are ways to regain Duality in the present system and a more thorough discussion of whether or not Duality is desirable is necessary, but beyond the scope of this paper.

W. STARR

$03 / 23 / 13$ 


\subsection{Dynamic Semantics and Counterfactual Expansion}

Why exactly did the analysis of conditionals presented above need a dynamic conception of meaning? The discussion in $\S \S 3.2$ and 3.3 gave some general motivation for a dynamic analysis by illustrating that it offers an interesting and powerful perspective on logic and communicative role of conditionals. But it is unclear that those benefits are exclusive to a dynamic approach, even if they are particularly natural from that perspective. As I mentioned at the end $\S 3.3$ there is a more explicit argument that can be made for a dynamic semantics and it hinges on the semantics of $\triangleleft$. Suppose one adopted a static strict conditional semantics.

\section{Static Strict Conditional $\llbracket($ if $\phi) \psi \rrbracket_{c}=\left\{w \in c \mid c \cap \llbracket \phi \rrbracket_{c} \subseteq \llbracket \psi \rrbracket_{c \cap \llbracket \phi \rrbracket_{c}}\right\}$}

The difference between subjunctive and indicative conditionals that needs to be captured is that subjunctives allow access to antecedent worlds outside $c$. This difference needs to come from the semantics of $\triangleleft$, which will occur somewhere in $\phi$. But there is a clear problem. No matter which proposition $\llbracket \phi \rrbracket$ turns out to be, $c \cap \llbracket \phi \rrbracket$ will be limited to $c$-worlds. So there is no propositional semantics that can be assigned to $\triangleleft \alpha$ such that (if $\triangleleft \alpha) \psi$ accesses $\alpha$-worlds outside $c$ but (if $\alpha$ ) $\psi$ accesses $\alpha$-worlds within $c$. The problem is obviously that the conditional semantics itself builds in the relativization to $c$ needed for indicative conditionals. An alternative approach is to begin with a conditional semantics that does not have this restriction:

Static Strict Conditional v2 $\llbracket($ if $\phi) \psi \rrbracket_{c}=\left\{w \in c \mid \llbracket \phi \rrbracket_{c} \subseteq \llbracket \psi \rrbracket_{\llbracket \phi \rrbracket_{c}}\right\}$

One could then claim indicatives contain an 'indicative mood operator' $\triangleright$ such that $\llbracket \triangleright \alpha \rrbracket_{c}=c \cap \llbracket \alpha \rrbracket$, while giving a semantics for $\triangleleft \alpha$ that can reach beyond $c$-worlds. Unfortunately, there is no morphological evidence for this indicative mood operator, let alone the cross-linguistic evidence justifying the $\triangleleft$ operator. Furthermore, as it stands the conditional propositions assigned by both static proposals here are very problematic. They are restricted to $c$-worlds, which means they are false everywhere outside $c$ and never provide information when asserted in $c$. This sits uncomfortably with the static picture and not even (if $\phi$ ) $\phi$ will come out as a logical truth. If this restriction is lifted, all conditionals become logical truths or falsities. By embracing a dynamic semantics for $\triangleleft$ one can satisfy these linguistic and logical constraints with 
ease. This is a powerful consideration in its favor.

\section{Disjunctive Antecedents and Reverse Sobel Sequences}

Here, I will use the analysis of subjunctive conditionals from $\S 2$ to shed light on two phenomena used recently to motivate a strict analysis of subjunctive conditionals: disjunctive antecedents and reverse Sobel sequences. In both cases, the discussion will have to be brief and preliminary. While the approach outlined below improves on existing ones, I will also mention some data that are not as easily explained by any existing account.

\subsection{Disjunctive Antecedents}

Many authors have noted that the Lewis-Stalnaker semantics yields an unsatisfying analysis of subjunctive conditionals with disjunctive antecedents. ${ }^{31}$ While the inference from (30a) to (30b) sounds impeccable, the Lewis-Stalnaker logic maintains that SDA is invalid.

(30) a. If Bob had danced or Sarah had sang, Andy would have cried

b. So, if Bob had danced, Andy would have cried, and if Sarah had sang, Andy would have cried

\section{Simplification of Disjunctive Antecedents (SDA)}

$$
\left(\phi_{1} \vee \phi_{2}\right)>\psi \vDash\left(\phi_{1}>\psi\right) \wedge\left(\phi_{2}>\psi\right)
$$

This is not a correctable quirk, but a consequence of the basic idea behind the Lewis-Stalnaker semantics. Suppose that the most similar $\phi_{1}$-worlds are $\psi$-worlds, but the most similar $\phi_{2}$-worlds are $\neg \psi$-worlds. Since every $\phi_{1}$-world is a $\phi_{1} \vee \phi_{2}$-world, it follows that $\left(\phi_{1} \vee \phi_{2}\right)>\psi$. SDA would allow it to follow that $\phi_{2}>\psi$, but by assumption $\phi_{2}>\neg \psi$.

Several responses to this problem have been explored in the literature. Some, like Nute (1975), Warmbrōd (1981a: §3) and Lycan (2001: 42-6), suggest changing or replacing the Lewis-Stalnaker semantics. Others, like Loewer (1976) and Nute (1980), try to address the problem pragmatically. As Warmbrōd (1981a) and Lycan (2001) observe, their strict-conditional accounts are by far the most

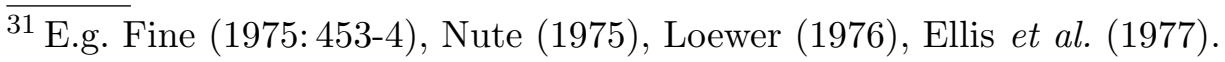

W. STARR

$03 / 23 / 13$ 
successful. Below I will show that the variably-strict analysis developed in $\S 2$ delivers an equally appealing analysis of disjunctive antecedents.

On the theory advanced in $\S 2,(30 \mathrm{a})$ corresponds to (if $(\triangleleft \mathrm{b} \vee \triangleleft \mathrm{s})$ ) a. The crucial point is that $\triangleleft$ occurs within each disjunct. Recall that the source of counterfactuality $\triangleleft$, has the syntax of a tense morpheme. As such, it does not take scope over compound sentences but rather attaches directly to a verb. Within a propositional formal language, the best one can do to model this is allowing $\triangleleft$ to attach to literals (atomics and negations thereof). Thus, a disjunctive antecedent does not concern the most similar $b \vee s$-worlds but rather the union - that is what $\vee$ does - of the most similar b-worlds and the most similar s-worlds. As a result, (if $(\triangleleft \mathrm{b} \vee \triangleleft \mathrm{s})$ ) a does not test that all of the most similar b $\vee$ s-worlds are a-worlds. Instead, it tests that among the most similar b-worlds and the most similar s-worlds there are only a-worlds. This clearly entails that the most similar b-worlds are a-worlds and that the most similar s-worlds are a-worlds, and hence that the inference in (30) is valid. Indeed, the logic that follows from the semantics in $\S 3.5$ delivers: ${ }^{32}$

Fact 2 (SDA is Valid) For any literals $\alpha$ and $\beta$

(if $(\triangleleft \alpha \vee \triangleleft \beta)) \psi \vDash(($ if $\triangleleft \alpha) \psi) \wedge(($ if $\triangleleft \beta) \psi)$ (proof in Appendix)

The problem of disjunctive antecedents thereby emerges as an artifact of representing the distinct morphological components of a subjunctive conditional as a single idiomatic connective, though two concerns still need to be addressed. ${ }^{33}$

McKay \& van Inwagen (1977) have claimed that there are counterexamples to SDA, since the inference in (31) sounds suspicious.

(31) a. If Spain had fought for the Axis or the Allies, she would have fought for the Axis

b. \# So, if Spain had fought for the Allies, she would have fought for the Axis

$\overline{32}$ More generally, if $\phi_{1}$ and $\phi_{2}$ are formulas built up from literals embedded under $\triangleleft$ by $\vee$ and $\wedge$, then (if $\left.\left(\phi_{1} \vee \phi_{2}\right)\right) \psi \vDash\left(\left(\right.\right.$ if $\left.\left.\phi_{1}\right) \psi\right) \wedge\left(\left(\right.\right.$ if $\left.\phi_{2}\right) \psi$ ).

33 Another raised by Fine (1975: 453-4) is that SDA leads to Antecedent Strengthening by the substitution of equivalents in the antecedent. $p$ and $(p \wedge q) \vee(p \wedge \neg q)$ are equivalent, so from $p>r,((p \wedge q) \vee(p \wedge \neg q))>r$ follows by substitution of equivalents. Then by SDA $(p \wedge q)>$ r. But $\triangleleft p$ and $\triangleleft((p \wedge q) \vee(p \wedge \neg q))$ are not equivalent.

W. STARR

$03 / 23 / 13$ 
But as Warmbrōd (1981a:284) notes, (31b) can be analyzed, like the alleged counterexamples discussed in $\S 3.2$, as a conditional with a failed presupposition. For (31a) to be accepted, there cannot be any accessible worlds in which Spain fought for the Allies. But then (31b) is bad because it fails to have its visibility presupposition (see §3.5) met. So embracing the validity of SDA under a Strawsonian definition of consequence is compatible with this example. This analysis is confirmed by the infelicity of (31a) after granting that Spain could have fought for the Allies:

(32) Spain didn't fight on either the Allied or Axis side, but she could have ended up with the Allies. \# Nevertheless, if she had fought for the Axis or the Allies, she would have fought for the Axis.

Yet more evidence can be extracted from the infelicity of (33).

(33) \# If Spain had fought for the Axis or if Spain had fought for the Allies, she would have fought for the Axis

The most plausible explanation of this is that both if-clauses carry a visibility presupposition. Presuppositions project through disjunction, so the complex antecedent has both visibility presuppositions. Since the second cannot met while making the conditional acceptable, the sentence is infelicitous. ${ }^{34}$

This analysis of (31) may seem to be in tension with the felicity of (34b), since it clearly admits of a possibility where Spain fought for the Allies. ${ }^{35}$

(34) a. If Spain had fought for the Axis or the Allies, she would have fought for the Axis

b. If Spain had fought for the Allies, she would have been obliterated by the Axis forces in Italy

$\overline{34}$ One might instead have the hunch that the difference between (33) and (31a) traces to the distinction between (if $\triangleleft(\alpha \vee \beta)$ ) $\psi$ and (if $\triangleleft \alpha \vee \triangleleft \beta) \psi$. But recall that $\triangleleft$ is coming from either the past tense or have. So one would expect If Spain had fourth for the Axis or Spain had fought for the Allies, she would have fought for the Axis to be just as bad as (33). Though it is subtle, this conditional strikes me as at least significantly better than (33). I return to $\triangleleft(\alpha \vee \beta)$ versus $\triangleleft \alpha \vee \triangleleft \beta$ shortly.

${ }^{35}$ I thank James Shaw for raising this issue.

W. STARR

$03 / 23 / 13$ 
However, this pattern can be accommodated by saying that a different $f$ is at work in (34b) and (34a). The question is then why we can switch to a different $f$ in (34b) but not in (31b). I believe the answer stems from the fact that (34b) occurs as an explanation of (34a) while (31b) occurs as the conclusion drawn from (31a). An inference requires $f$ to remain constant to avoid equivocation. But explanation needed have that structure. One can easily view (34b) as explaining why an $f$ which admits the possibility of Spain fighting for the Allies is excluded: Spain would have been obliterated. As such, (34b) should not be seen as exploiting the same selection function as (34a), but rather justifying the kind of selection function needed to make (34a) come out true.

A lingering concern is that the problem of SDA has been solved by unjustifiable syntactic fiat. While (35) is predicted, (36) is not for the same reasons that Lewis and Stalnaker do not predict SDA.

(35) (if $\triangleleft \mathrm{A} \vee \triangleleft \mathrm{B}) \mathrm{C} \vDash($ if $\triangleleft \mathrm{A}) \mathrm{C} \wedge$ (if $\triangleleft \mathrm{B}) \mathrm{C}$

(36) (if $\triangleleft(A \vee B)) C \vDash($ if $\triangleleft A) C \wedge$ (if $\triangleleft B) C$

Am I just denying that disjunctive antecedents are ever represented as $\triangleleft(\mathrm{A} \vee$ B)? But surely this is a plausible representation for the conditionals in (37) where disjunction appears under $\triangleleft$.

(37) a. If Kathy or Grace had attended my talk, I would have been refuted

b. $X$ : Bill saw a bear or a coyote.

$Y$ : No, if that were true, he would have taken a picture.

After all, or in the antecedent of (37a) plausibly scopes under past tense and have. Modal anaphora to a disjunctive proposition seems to accomplish the same thing in (37b). It is possible, however, to render $\triangleleft(\alpha \vee \beta)$ and $\triangleleft \alpha \vee \triangleleft \beta$ equivalent. One way is to adopt an alternative semantics for disjunction (Aloni 2003; Simons 2005). The basic idea is that disjunctions do not present a single disjunctive proposition, but rather a set of propositions; one for each disjunct. The $\triangleleft$ operator can then be made to distribute over each proposition in this set. This proposal is formalized in the appendix (see Definition 24 and Remark 5 ), where it is also proven that it renders (if $\triangleleft(\alpha \vee \beta)$ ) $\psi$ and (if $\triangleleft \alpha \vee \triangleleft \beta$ ) $\psi$ are equivalent. ${ }^{36}$ However, this analysis now seems to me insufficiently general,

$\overline{36}$ Alonso-Ovalle $(2006,2009)$ also presents a (more complex) analysis using alternative semantics. This analysis does not use an operator like $\triangleleft$ to apply the selection

W. STARR

$03 / 23 / 13$ 
since negated conjunctions give rise to the same issue (Nute 1980: 33). ${ }^{37}$

(38) a. If Nixon and Agnew had not both resigned, Ford would never have become President

b. If Nixon had not resigned, Ford would never have become President

c. If Agnew had not resigned, Ford would never have become President

(39) a. If Elvis hadn't drank and smoked, he would have lived longer

b. If Elvis hadn't drank, he would have lived longer

c. If Elvis hadn't smoked, he would have lived longer

In both cases, (b) and (c) intuitively follow from (a). In the successful analysis of narrow scope disjunctive antecedents (where $\triangleleft$ scopes under $\vee$ ), alternatives were not necessary. Why did this analysis work, and why doesn't this success extend to wide scope disjunctive antecedents (where $\triangleleft$ scopes over $\vee$ ?) The analysis worked because disjunction unions two updates, each of which has $\triangleleft$ being applied to that disjunct. Furthermore, since each antecedent in the conclusion of SDA carries a visibility presupposition, each case where $\triangleleft$ is applied to a disjunct in the premise must yield a non-empty set of worlds. However, when $\triangleleft$ takes wide scope, it does not take into account the structure of the updates induced by its scope. Instead of defining $c_{f}[\triangleleft \alpha]$ in terms of $[\alpha]$, it was defined in terms of $\llbracket \alpha \rrbracket$. Although the latter set of worlds is determined by $[\alpha],[\alpha]$ itself has more structure when $\alpha$ contains connectives. For example, $[\mathrm{A} \vee \mathrm{B}]$ involves merging the parallel processing of A and B. If $c_{f}[\triangleleft \alpha]$ could be defined to exploit this kind of structure, say to apply an expansion to subupdates, I believe a general solution could be arrived at. It is tricky in the

$\overline{\text { function }}$ to alternatives. It instead builds an appeal to the selection function into the basic conditional semantics. This will not lead to an adequate uniform theory of conditionals for the reasons discussed in $\S 2.3$. The lack of $\triangleleft$ and dynamic consequence in this analysis also prevents it from having a compelling response to worries about the substitution of equivalents in antecedents (see note 33).

${ }^{37}$ Fine (2012b: 47) also voices this worry, and additionally mentions examples with existential quantification as a worry. Since many also adopt an alternative semantics for existential quantification, that additional worry is mute. Fine $(2012 \mathrm{a}, \mathrm{b})$ offers a different semantics for subjunctive conditionals altogether that validates SDA. Fine (2012b: 48) sketches an inchoate version of the Warmbrōd (1981a:284)-style reply to McKay \& van Inwagen (1977) detailed above. The Fine (2012a) semantics shares many similarities to my preferred approach mentioned above (Starr 2013b), but does not integrate well into the kind of uniform theory developed here.

W. STARR

$03 / 23 / 13$ 
current setting to make this work for negation, but with additional resources it may be possible. This project is taken up in Starr (2013b) where I develop a semantics based on structural equations rather than comparative similarity.

\subsection{Reverse Sobel Sequences}

Dynamic strict conditional analyses have been developed to explain the contrast in (40) and (41) between Sobel sequences and reverse Sobel Sequences (von Fintel 2001; Gillies 2007).

(40) a. If Sophie had gone to the parade, she would have seen Pedro dance

b. But of course, if Sophie had gone to the parade and been stuck behind someone tall, she would not have seen Pedro dance

(41) a. If Sophie had gone to the parade and been stuck behind someone tall, she would not have seen Pedro dance

b. \# But of course, if Sophie had gone to the parade, she would have seen Pedro dance

However, the dynamic variably-strict analysis offered here offers a different explanation of this contrast. This analysis proposes that this is an instance of modal subordination, a phenomena where modals and pronouns are interpreted against a salient body of information other than $c$ (Roberts 1989; Frank \& Kamp 1997; Stone 1999). Consider the pronoun it in (42c).

(42) a. Your cabin wasn't raided by a coyote.

b. But if a coyote had raided your cabin, it would've eaten your dinner.

c. It would have eaten your meat first.

This anaphoric element requires (42c) to be interpreted against a background where there is a coyote who entered your cabin and ate your dinner. Since (42a) eliminates any such worlds from $c, c$ cannot be this background. A natural idea is that (42b) introduces a counterfactual set of worlds where a coyote raided your cabin and eats your dinner, call it $r$. The would in (42c) helps indicate that the sentence is to be interpreted against a salient set of worlds other than $c$. In this example, $r$ is the only alternative. To fill out this story, it is necessary to have a model of information that explicitly encodes eligible referents for pronouns, i.e. an assignment function. But this is not necessary for 
my purposes. I just wish to claim that conditionals introduce hypothetical sets of worlds other than $c$, and that these too are recorded in context. Adapting Kaufmann (2000), I proposed just such a theory in Starr (2011) to capture the fact that if simultaneously functions in conditionals and embedded questions. Abstracting away from the need for $C_{f}$ rather than $c_{f}$, the idea is that sentences do not merely operate on $c_{f}$, but on a sequence consisting of $c_{f}$ plus additional, hypothetical states, $\left\langle c_{f},\left\langle i_{f^{\prime}}, \cdots\right\rangle\right\rangle .{ }^{38}$ The information being taken for granted

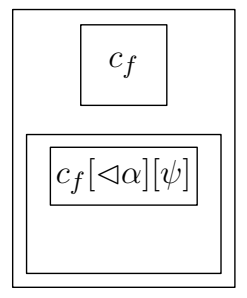

Fig. 1. The Result of a Successful Update with (if $\triangleleft \alpha) \psi$

(c) is always the leftmost element. Successfully updating with conditionals like (42a), represented (if $\triangleleft \mathrm{c}$ ) d, leave $c_{f}$ as it was, having guaranteed that all of the most similar coyote-raiding-your-cabin worlds are coyote-ate-your-dinner worlds. However, the subordinate context $c_{f}[\triangleleft \mathrm{c}][\mathrm{d}]$ generated by that update remains in the output state of that update. (42c) supports this analysis, since it anaphorically targets the subordinate context. While a normal update with a subsequent sentence like (42c) targets $c_{f}$, there is a different way of relating the assertion of $\mathrm{m}$ to the context at work here. Here, the subordinate context $c_{f}[\triangleleft \mathrm{c}][\mathrm{d}]$ gets tested but the result of this test has implications for $c_{f}$ as well. I called this kind of update Conclusion in Starr (2011), since it is akin to drawing a conclusion at the end of a line of suppositional reasoning. Such an act relates an assertion to a hypothetical body of information, but does so in a way that has implications for the information actually being taken for granted. The following definition characterizes this operation.

Definition 11 (Conclusion) Where $s=\left\langle c_{f},\left\langle c_{f_{0}}^{0}, \ldots\left\langle c_{f_{n}}^{n}\right\rangle \cdots\right\rangle\right\rangle$ :

$s \uparrow \psi=\left\langle\left\{w \in c \mid\left\langle c_{f_{n}}^{n}\right\rangle \vDash \psi\right\}_{f},\left\langle c_{f_{0}}^{0}, \ldots\left\langle c_{f_{n}}^{n},\left\langle c_{f_{n}}^{n}\right\rangle[\psi]\right\rangle \cdots\right\rangle\right\rangle$

$\overline{38}\left\langle c_{f}\right\rangle$ is a state and if $s$ is a state then $\left\langle c_{f^{\prime}}^{\prime}, s\right\rangle$ is a state. This recursive structure allows one meaning for $\phi$ to be applied to both $\left\langle c_{f},\left\langle c_{f^{\prime}}^{\prime}\right\rangle\right\rangle$ and it's sub-state $\left\langle c_{f^{\prime}}^{\prime}\right\rangle$. This is needed for conditionals in the consequent of a conditional.

W. STARR

$03 / 23 / 13$ 
On this analysis the intuitive interpretation of (42) is $s[\neg \mathrm{c}][($ if $\triangleleft \mathrm{c}) \mathrm{d}] \uparrow \mathrm{m}$, not $s[\neg \mathrm{c}][($ if $\triangleleft \mathrm{c}) \mathrm{d}][\mathrm{m}]$. While the latter is contradictory the former is flawless.

More broadly construed, the idea is that language users track different bodies of information. The intended structure of a given discourse may relate subsequent utterances to the contextual possibilities $\left(c_{f}\right)$ or the most recently introduced hypothetical possibilities $\left(c_{f_{n}}^{n}\right)$. Understanding when a speaker is relating them one way rather than another will combine linguistic cues, e.g. would, with contextual information, e.g. $\neg \mathrm{C}$, and general principles of practical rationality. This general way of thinking about the structure of discourse yields a compelling account of modal subordination. I believe it also explains the contrast between Sobel sequences and their reversed counterparts.

Formalized, the Sobel sequence (40) comes out as (43) and it's reversed counterpart (41) comes out as (44).

(43) a. (if $\triangleleft s) d$

b. $\quad($ if $\triangleleft s \wedge \triangleleft t) \neg d$

(44) a. (if $\triangleleft s \wedge \triangleleft t) \neg d$

b. \# (if $\triangleleft \mathrm{s}) \mathrm{d}$

According to the semantics advanced above both $s[$ (if $\triangleleft \mathrm{s}) \mathrm{d}][($ if $\triangleleft \mathrm{s} \wedge \triangleleft \mathrm{t}) \neg \mathrm{d}]$ and $s[($ if $\triangleleft \mathrm{s} \wedge \triangleleft \mathrm{t}) \neg \mathrm{d}][($ if $\triangleleft \mathrm{s}) \mathrm{d}]$ are consistent updates. So the semantics by itself does not account for the asymmetry. However, once an alternative structure is countenanced for discourse (44), namely $s[$ (if $\triangleleft \mathrm{s} \wedge \triangleleft \mathrm{t}) \neg \mathrm{d} \uparrow$ (if $\triangleleft \mathrm{s}$ ) d, an alternative account is possible. The sequence of changes imposed by this structure does yield inconsistency. On this analysis, this discourse tests whether $c_{f}[\triangleleft \mathrm{s}][\triangleleft \mathrm{t}][\neg \mathrm{d}][\triangleleft \mathrm{s}] \vDash \mathrm{d}$. $c_{f}[\triangleleft \mathrm{s}]$ will deliver a set of s-worlds, some of which may be outside $c_{f} . c_{f}[\triangleleft \mathrm{s}][\triangleleft \mathrm{t}]$ will refine that set to the $\mathbf{s} \wedge \mathrm{t}$-worlds. Since $c_{f}$ was successfully updated with (44a), these $s \wedge t$-worlds must be $\neg$ d-worlds. So updating this set with $\neg$ d has no effect. Updating this set with $\triangleleft$ s will also be ineffectual, since all the worlds in that set are already s-worlds. Clearly this set does not support $d$ since every world in it is a $\neg$ d world. Hence, the test imposed by (44) is failed and $c_{f}$ is reduced to $\varnothing$, the contradictory body of information. That is why (44) is infelicitous. By contrast, (43) is consistent no matter how its discourse structure is interpreted.

W. STARR

$03 / 23 / 13$ 
There is additional support for this approach to reverse Sobel sequences. The contrast between Sobel sequences and their reversals becomes much sharper when the discourse structure is made explicit with anaphoric pronouns.

(45) a. If Sophie had bought a cupcake, she would have eaten it

b. But of course, if Sophie had bought it but then dropped it in the sewer, she would not have eaten it

(46) a. If Sophie had bought a cupcake but then dropped it in the sewer, she would not have eaten it

b. \# But of course, if Sophie had bought it, she would have eaten it

The occurrences of it in (45b) must be modally subordinated to the hypothetical context set up by (45). This requires a discourse structure where the sentences are not simply updated in sequence, but one like Conclusion where the second sentence targets the hypothetical context.

There is a pressing question that this approach must address. If there are two potential discourse structures that can be read into reverse Sobel sequences, why do we so naturally fall into the subordination-style interpretation, particularly in cases like (41) where the alternative interpretation is felicitous? Although there are two structures that can be assigned to a discourse like (41), they are not equally plausible. When a possibility involving Sophie going to the parade is evoked and a subsequent sentence also makes reference to such a possibility, there is a strong presumption that the second sentence is commenting on the topic raised by the first. I take this to be a clear truth about the evolution of discourse, but one which only a far more sophisticated theory and discussion of discourse structure could predict. Unless there is something to explicitly defeat this presumption, it will be very difficult to assign a structure to (45) where the sentences are simply updated in sequence. But as (Moss to appear: §4) details, there are such cases. Moss imagines a case where the speaker wants to indirectly convey the information that Mary would have turned down a marriage proposal from John.

(47) a. If John had proposed to Mary and she had said yes, he would have been really happy

b. But if John had proposed to Mary, he would have been really unhappy

W. STARR

$03 / 23 / 13$ 
The antecedents in (47) must be about different scenarios. The second scenario of John proposing can't be one where Mary says yes. So (47b) is not being interpreted against the hypothetical possibilities set up by (47) but just the contextual possibilities. This is evidenced in the drastic failure of anaphorically linking a parallel pair of conditionals.

(48) Although John was seriously considering a proposal to Mary, he didn't end up proposing. He never even bought a ring.

(49) a. If John had offered Mary an engagement ring and she had said yes, he would have been really happy

b. \# But if John had offered it to Mary, he would have been really unhappy

While (47) is a surprise for the von Fintel-Gillies theories, (49) is a surprise for Moss's approach, which is built on a generalization about when it is epistemic responsibility to utter certain sentences. The basic idea is that it is epistemically irresponsible to assert propositions that are incompatible with salient possibilities the agent cannot rule out. This disallows (41) but allows (47) since the speaker can rule out the possibility that John will proposed to Mary and she will say yes. But by the same lights, one should be able to utter (49b). Both cases can be handled by the approach here, though I must admit that more needs to be said about the theory of discourse structure before victory is declared. I simply wish to mention here that the present analysis of subjunctive conditionals plus independently motivated assumptions about the structure of discourse and modal subordination suffices to explain the phenomena of reverse Sobel sequences. ${ }^{39}$

\section{Conclusion}

This paper has developed and unified ideas a wide range of ideas:

(a) subjunctive antecedents contain a modal operator (Isard 1974; Lyons 1977; Iatridou 2000; Schulz 2007: Ch.6)

$\overline{39}$ Like von Fintel's theory, the apparatus here also allows one to explain the intuitive validity of many cases of transitivity as well as the asymmetries those inferences exhibit, but that too must be reserved for another occasion.

W. STARR

$03 / 23 / 13$ 
(b) subjunctive antecedents concern worlds that may be outside $c$ (Stalnaker 1975; von Fintel 1999b)

(c) indicatives presuppose their antecedent is compatible with $c$ (Stalnaker 1975; Adams 1975b; Gillies 2009)

(d) attending to the felicity conditions of conditionals impacts the debate about their logic Warmbrōd (1981b); Veltman (1986); Gillies (2009)

(e) understanding the interaction of the modal operator in subjunctive antecedents is the key to understanding disjunctive antecedents (Here); alternative semantics may help some (Alonso-Ovalle 2009)

(d) sequences of conditionals involve modal anaphora (Stone 1999; Brasoveanu 2010)

Previous work has either offered no formal semantics to go with (a), has not unified that semantics with (b) or shown how it could fit into a theory that accepts (c) and (d). As was argued above, alternative semantics is not enough for (e). It must be combined at least with (a) to yield a compelling account of disjunctive antecedents. No existing approach has attempted to unify Sobel sequences with (d). While I have significantly developed many of these ideas here, the main achievement is their smooth integration into one simple and general picture about the semantics of conditionals.

The semantics for $\triangleleft$ encoded the idea that subjunctive, unlike indicative, conditionals can involve a revision of an agent's information. However, it is quite important to qualify by noting that this kind of revision does not seem to be the same kind of revision we engage in when we learn our beliefs are false. Suppose you've just come into your office, flipped the switch up and the light is now on. You'd obviously accept (50).

(50) If this switch were down, the light would be off

Yet, if you turned around and saw that the light switch was actually down, you wouldn't come to accept the claim that the light is off. You'd more likely believe the light switch was inoperative and a motion detector had been installed; or maybe that your office is haunted. What these kind of examples illustrate is that subjunctive conditionals seem to provide a very local manipulation of a particular fact while general belief revision is a more global process. A semantics for $\triangleleft$ based on manipulating structural equations (Pearl 2000; Thomason 2007) is a promising way to respect this distinction while

W. Starr

$03 / 23 / 13$ 
preserving the analysis sketched above.

Section $\S 4$ vindicated a variably-strict analysis in the face of some of the most important empirical challenges it has encountered. In terms of adjudicating between strict and variably-strict analyses this is an interesting result but as said in the introduction, my motivations are more general. I believe that $f$ should not be analyzed in terms of similarity, but rather in terms of dependence, using the framework of structural equations. I think that only this approach can draw the deep connections between counterfactuals, belief revision and the structure of the natural world that have spurred their investigation in the philosophy of science and artificial intelligence. Like the similarity analysis, this will result in a variably-strict theory. By addressing some of the empirical problems for that approach, I hope to have brought us one step closer to addressing the questions about subjunctive conditionals that have made them a persistent interest of philosophers and cognitive scientists.

This paper has illustrated a fruitful application of a dynamic approach to semantics where the meaning of a morpheme is not to be identified with its content (or even its character). Its meaning is the characteristic effect it has on the mental states of language users. Sentences can be associated with contents, since these states have contents. But the fundamental notions of semantics word meaning, compositionality, entailment and veracity (truth or support) are richer than a content-based theory can capture. It is always appropriate to regard new approaches critically and cautiously. But, I hope this work will encourage other philosophers to explore the resources provided by dynamic approaches and seriously consider it as not just a different technical apparatus, but a different and interesting perspective in the theory of meaning.

\section{Acknowledgements}

I benefitted greatly from participants in my Spring 2012 graduate seminar at Cornell and from audiences at NYU and the University of Michigan Philosophy and Linguistics Workshop. The basic ideas here come from my dissertation (Starr 2010: Ch.3), so the lengthy acknowledgments there apply again here. I owe a special debt to conversations with Barbara Abbott, Ana Arregui, Josh Armstrong, Maria Bittner, Carlos Fasola, Thony Gillies, Gabe Greenberg, Michael Johnson, Jeff King, Philipp Koralus, Ernie Lepore, Karen Lewis, Barry Loewer, Salvador Mascarenhas, Sarah 
Murray, Carlotta Pavese, Adam Rigoni, Bob Stalnaker, Jason Stanley, James Shaw, Matthew Stone, Rich Thomason, Brian Weatherson and Malte Willer.

\section{A Dynamic Conditional Logic with $\triangleleft\left(\mathrm{DCL}_{\triangleleft}\right)$}

\section{A.1 Syntax and Semantics}

\section{Definition $12\left(\mathrm{DCL}_{\triangleleft}\right.$ Syntax)}

(5) $(\phi \wedge \psi) \in \mathcal{W} f f_{A} \quad$ if $\phi, \psi \in \mathcal{W}_{f f}$

(6) $(\phi \vee \psi) \in{\mathcal{W} f f_{A}}$ if $\phi, \psi \in \mathcal{W}_{f f}$

(7) (if $\phi) \psi \in \mathcal{W} f f_{C} \quad$ if $\phi, \psi \in \mathcal{W} f f_{A}$

(8) (if $\phi) \psi \in \mathcal{W} f f_{C} \quad$ if $\phi \in \mathcal{W}_{f f}, \psi \in \mathcal{W} f f_{C}$

(9) $\quad \neg \phi \in \mathcal{W} f f_{C}$ if $\phi \in \mathcal{W} f f_{C}$

(10) $\nabla \phi \in \mathcal{W} f f_{C}$ if $\phi \in \mathcal{W} f f_{C}$

(11) $\square \phi \in \mathcal{W} f f_{C}$ if $\phi \in \mathcal{W} f f_{C}$

(12) $(\phi \wedge \psi) \in \mathcal{W} f f_{C} \quad$ if $\phi, \psi \in \mathcal{W}_{f f_{C}} \cup \mathcal{W} f f_{A}$

$(13)(\phi \vee \psi) \in \mathcal{W} f f_{C} \quad$ if $\phi, \psi \in \mathcal{W}_{f f_{C}} \cup \mathcal{W} f f_{A}$

(14) $\quad \forall \phi \in \mathcal{W} f f_{A \triangleleft} \quad$ if $\phi \in \mathcal{W} f f_{A}$

(15) $\phi_{1} \vee \phi_{2} \in \mathcal{W} f f_{A \triangleleft} \quad$ if $\phi_{1}, \phi_{2}, \in \mathcal{W} f f_{A \triangleleft}$

(16) $\phi_{1} \wedge \phi_{2} \in \mathcal{W} f f_{A \triangleleft} \quad$ if $\phi_{1}, \phi_{2}, \in \mathcal{W} f f_{A \triangleleft}$

(17) (if $\phi) \psi \in \mathcal{W} f f_{\triangleleft} \quad$ if $\phi \in \mathcal{W}_{f f_{A \triangleleft}}, \psi \in \mathcal{W} f f_{A}$

(18) $\square \triangleleft \phi \in \mathcal{W} f f_{\triangleleft}$ if $\phi \in{\mathcal{W} f f_{A}}$

(19) $\diamond \triangleleft \phi \in \mathcal{W} f f_{\triangleleft}$ if $\phi \in \mathcal{W} f f_{A}$

(20) (if $\phi) \psi \in \mathcal{W} f f_{\triangleleft} \quad$ if $\phi \in \mathcal{W} f f_{A \triangleleft}, \psi \in \mathcal{W} f f_{\triangleleft}$

(21) $\quad \neg \phi \in \mathcal{W} f f_{\triangleleft}$ if $\phi \in \mathcal{W} f f_{\triangleleft}$

(22) $(\phi \wedge \psi) \in \mathcal{W} f f_{\triangleleft} \quad$ if $\phi, \psi \in \mathcal{W}_{f f_{C}} \cup \mathcal{W} f f_{A} \cup \mathcal{W} f f_{\triangleleft}$

(23) $(\phi \vee \psi) \in \mathcal{W} f f_{\triangleleft} \quad$ if $\phi, \psi \in \mathcal{W}_{f f_{C}} \cup \mathcal{W} f f_{A} \cup \mathcal{W} f f_{\triangleleft}$

W. STARR

$03 / 23 / 13$ 


\section{A.1 Syntax and Semantics}

Remark 1 This does not permit indicative conditionals to nest in subjunctive conditionals or vice-versa, though that could be easily achieved. This also prohibits any conditional from nesting in the antecedent of a conditional. This too could be permitted, but seems unmotivated by natural language syntax.

Definition 13 (Worlds) $W: \mathcal{A} t \mapsto\{1,0\}$ where $\mathcal{A} t=\left\{\mathrm{p}_{0}, \mathrm{p}_{1}, \ldots\right\}$

Definition 14 (Contextual Possibilities/Information) $c \subseteq W$

\section{Definition 15 (Contextual Alternatives)}

- $C$ is a non-empty set of subsets of $W$ - $\varnothing \neq C \subseteq \mathscr{P}(W)$

- $C$ is the set of all such $C$

- $\cup C$ is the information embodied by $C$; the sets in $C$ are called alternatives; overlapping and non-maximal alternatives are allowed.

Definition 16 (Selection Functions) (Let $p, p^{\prime} \subseteq W$ and $w \in W$ )

(a) $f(w, p) \subseteq p$ success

(b) $f(w, p)=\{w\}$, if $w \in p$ strong centering

(c) $\quad f(w, p) \subseteq p^{\prime} \& f\left(w, p^{\prime}\right) \subseteq p \Longrightarrow f(w, p)=f\left(w, p^{\prime}\right)$ uniformity

(d) $f(w, p)$ contains at most one world uniqueness

- While Stalnaker assumes (d), I will not.

\section{Definition 17 (Contexts)}

- If $C$ is a set of contextual alternatives and $f$ is a selection function, then $\langle C, f\rangle$ is a context.

- Nothing else is a context

Remark $2\left(C_{f}\right) \quad C_{f}$ is an abbreviation for $\langle C, f\rangle$, and any set theoretic notation involving $C_{f}$ should be read as operating on the $C$ inside $C_{f}$. E.g. $C_{f} \cap C_{f}^{\prime}$ is an abbreviation for $\left\langle C \cap C^{\prime}\right\rangle_{f}$. This notation will never be used in a case like $C_{f} \cap C_{f^{\prime}}^{\prime}$ if $f \neq f^{\prime}$. 


\section{A.1 Syntax and Semantics}

\section{Definition 18 (Update Semantics)}

Where $C=\left\{c_{0}, \ldots, c_{n}\right\}$ and $\bar{C}^{\phi}:=\left\{c_{0}-\bigcup\left(\left\{c_{0}\right\}[\phi]\right), \ldots, c_{n}-\bigcup\left(\left\{c_{n}\right\}[\phi]\right)\right\}$ :

$$
\begin{aligned}
C_{f}[\mathrm{p}] & =\left\{\left\{w \in c_{0} \mid w(\mathrm{p})=1\right\}, \ldots,\left\{w \in c_{n} \mid w(\mathrm{p})=1\right\}\right\}_{f} \\
C_{f}[\neg \phi] & =\bar{C}_{f}^{\phi} \\
C_{f}[\phi \wedge \psi] & =\left(C_{f}[\phi]\right)[\psi] \\
C_{f}[\phi \vee \psi] & =C_{f}[\phi] \cup C_{f}[\psi]
\end{aligned}
$$

Remark 3 Above, $\bar{C}^{\phi}$ may be pronounced the $\phi$ complement of $C$. Forming this set amounts to eliminating the $\phi$-worlds from each alternative in $C$. So $C_{f}[\neg \phi]$ will eliminate $\phi$-worlds from each alternative in $C$.

Definition 19 (Support, Truth in $w$ )

(1) Support $C_{f} \vDash \phi \Leftrightarrow \cup\left(C_{f}[\phi]\right)=\bigcup C_{f}$

(2) Truth in $w, f w, f \vDash \phi \Leftrightarrow\{\{w\}\}_{f}[\phi]=\{\{w\}\}_{f}$

Definition 20 (Propositional Content) $\llbracket \phi \rrbracket_{f}=\{w \mid w, f \vDash \phi\}$

Definition 21 (Inquisitive Content) $\lfloor\phi\rfloor_{f}=C^{\prime} \Longleftrightarrow\{W\}_{f}[\phi]=\left\langle C^{\prime}, f\right\rangle$

Remark 4 Since $\triangleleft$ is the only operator sensitive to $f$, I will often omit $f$ when writing $\lfloor\phi\rfloor$ and $\llbracket \phi \rrbracket$ for $\phi$ that do not contain it.

Definition 22 (Entailment) $\phi_{1}, \ldots, \phi_{n} \vDash \psi \Leftrightarrow \forall C_{f}: C_{f}\left[\phi_{1}\right] \cdots\left[\phi_{n}\right] \vDash \psi$

- If $C_{f}\left[\phi_{1}\right] \cdots\left[\phi_{n}\right][\psi]$ is defined.

Definition 23 (Conditional Semantics)

$C_{f}[($ if $\phi) \psi]= \begin{cases}\left\{a \in C \mid C_{f}[\phi] \vDash \psi\right\}_{f} & \text { if } \bigcup\left(C_{f}[\phi]\right) \neq \varnothing \\ \text { Undefined } & \text { otherwise }\end{cases}$

Definition 24 (Alternative Counterfactual Expansion $\triangleleft$ )

Let $f$ be a selection function and $c=\cup C$ :

$C_{f}[\triangleleft \alpha]=\left\{\left\{w^{\prime} \mid \exists w \in c: w^{\prime} \in f(w, a)\right\} \mid a \in\lfloor\alpha\rfloor\right\}_{f}$ 


\section{A.1 Syntax and Semantics}

Remark 5 To illustrate the definition, consider $C_{f}[\triangleleft \mathrm{A}]$ :

$$
\begin{aligned}
C_{f}[\triangleleft \mathrm{A}] & =\left\{\left\{w^{\prime} \mid \exists w \in c: w^{\prime} \in f(w, a)\right\} \mid a \in\lfloor\mathrm{A}\rfloor\right\}_{f} \\
& =\left\{\left\{w^{\prime} \mid \exists w \in c: w^{\prime} \in f(w, \llbracket \mathrm{A} \rrbracket)\right\}\right\}_{f}
\end{aligned}
$$

The second line follows since $\lfloor A\rfloor=\{\llbracket A \rrbracket\}$. By the meaning of $\vee$, $C_{f}[\triangleleft \mathrm{A} \vee \triangleleft \mathrm{B}]=C_{f}[\triangleleft \mathrm{A}] \cup C_{f}[\triangleleft \mathrm{B}]$. From the previous example it should be clear that this results in (A.1), the set of alternatives which were previously unioned in (??).

(A.1) $\left\{\left\{w^{\prime} \mid \exists w \in c: w^{\prime} \in f(w, \llbracket \mathrm{A} \rrbracket)\right\},\left\{w^{\prime} \mid \exists w \in c: w^{\prime} \in f(w, \llbracket \mathrm{B} \rrbracket)\right\}\right\}_{f}$ As desired, $C_{f}[\triangleleft(\mathrm{A} \vee \mathrm{B})]$ will yield the same result.

$$
\begin{aligned}
C_{f}[\triangleleft(\mathrm{A} \vee \mathrm{B})] & =\left\{\left\{w^{\prime} \mid \exists w \in c: w^{\prime} \in f(w, a)\right\} \mid a \in\lfloor\mathrm{A} \vee \mathrm{B}\rfloor\right\}_{f} \\
& =\left\{\left\{w^{\prime} \mid \exists w \in c: w^{\prime} \in f(w, \llbracket \mathrm{A} \rrbracket)\right\},\left\{w^{\prime} \mid \exists w \in c: w^{\prime} \in f(w, \llbracket \mathrm{B} \rrbracket)\right\}\right\}_{f}
\end{aligned}
$$

In this case, the second line follows since $\lfloor A \vee B\rfloor=\{\llbracket A \rrbracket, \llbracket B \rrbracket\}$. Thus, evaluating a disjunctive antecedent comes to the same thing whether the disjunction takes wide or narrow scope. It comes to evaluating whether or not the expanded set of alternatives supports the consequent. This raises a pertinent question: I've said what it takes for a set of worlds to support a sentence, but what does it take for a set of alternatives to support a sentence? Requiring that $C[\phi]=C$ produces an interesting but more fine-grained notion of support, requiring not only that $\phi$ provided no new information, but also introduced no new alternatives. To capture the old notion of support, I want to say that the underlying set of worlds is the same in $C$ as in $C[\phi]$. This is accomplished in Definition 22. 


\section{A.2 Results}

\section{A.2 Results}

Fact 3 (Simple Subjunctive Conditional Propositions)

For $\psi \in \mathcal{W} f f_{A}$ and free of $\diamond$ and $\square$, where $\lfloor\alpha\rfloor=\left\{a_{0}, \ldots, a_{n}\right\}$ :

(1) $\llbracket($ if $\triangleleft \alpha) \psi \rrbracket_{f}$ is well-defined iff $\forall w \in W, \exists a \in\lfloor\alpha\rfloor: f(w, a) \neq \varnothing$

By Def's 20, 19.2, 23 and $24, \quad \llbracket($ if $\triangleleft \alpha) \psi \rrbracket_{f}=\{w \quad \mid$ $\left.\left\{f\left(w, a_{0}\right), \ldots, f\left(w, a_{n}\right)\right\}_{f} \vDash \psi\right\}$ when defined. By Def 23, this is defined if and only if for all $w \in W, \bigcup\left\{f\left(w, a_{0}\right), \ldots, f\left(w, a_{n}\right)\right\} \neq \varnothing$. This holds at an arbitrary $w$ iff $\exists a \in\lfloor\alpha\rfloor: f(w, a) \neq \varnothing$.

(2) When defined, 【(if $\triangleleft \alpha) \psi \rrbracket_{f}=\left\{w \mid \bigcup\left\{f\left(w, a_{0}\right), \ldots, f\left(w, a_{n}\right)\right\} \subseteq \llbracket \psi \rrbracket_{f}\right\}$

As previously noted, 【(if $\triangleleft \alpha) \psi \rrbracket_{f}=\left\{w \mid\left\{f\left(w, a_{0}\right), \ldots, f\left(w, a_{n}\right)\right\}_{f} \vDash \psi\right\}$. $\psi$ is neither conditional nor modal, so $\forall C_{f}: C_{f} \vDash \psi$ iff $\cup C \subseteq \llbracket \psi \rrbracket_{f}$.

Fact 4 (Import-Export) (if $\left.\phi_{1}\right)\left(\left(\right.\right.$ if $\left.\left.\phi_{2}\right) \psi\right)=\vDash\left(\right.$ if $\left.\phi_{1} \wedge \phi_{2}\right) \psi$

PROOF When both forms are defined, they amount to the same update:

$$
\begin{aligned}
C_{f}\left[\left(\text { if } \phi_{1}\right)\left(\left(\text { if } \phi_{2}\right) \psi\right)\right] & =\left\{a \in C_{f} \mid C_{f}\left[\phi_{1}\right] \vDash\left(\text { if } \phi_{2}\right) \psi\right\}_{f} \\
& =\left\{a \in C_{f} \mid \bigcup\left(C_{f}\left[\phi_{1}\right]\left[\left(\text { if } \phi_{2}\right) \psi\right]\right)=\bigcup\left(C_{f}\left[\phi_{1}\right]\right)\right\}_{f} \\
& =\left\{a \in C_{f} \mid \bigcup\left\{a^{\prime} \in C_{f}\left[\phi_{1}\right] \mid C_{f}\left[\phi_{1}\right]\left[\phi_{2}\right] \vDash \psi\right\}=\bigcup\left(C_{f}\left[\phi_{1}\right]\right)\right\}_{f} \\
& =\left\{a \in C_{f} \mid C_{f}\left[\phi_{1}\right]\left[\phi_{2}\right] \vDash \psi\right\}_{f} \\
& =\left\{a \in C_{f} \mid C_{f}\left[\phi_{1} \wedge \phi_{2}\right] \vDash \psi\right\}_{f} \\
& =C_{f}\left[\left(\text { if } \phi_{1} \wedge \phi_{2}\right) \psi\right]
\end{aligned}
$$

Cases of undefinedness do not count towards validity and may be disregarded.

Fact 5 (Modus Ponens) (if $\phi) \psi, \phi \vDash \psi$

Proof Either $C_{f}[($ if $\phi) \psi]=C_{f}$ or $C_{f}[($ if $\phi) \psi]=\varnothing_{f}$. In the former case, $C_{f}[\phi] \vDash \psi$ and the condition needed for the entailment, $\bigcup\left(C_{f}[(\right.$ if $\left.\phi) \psi][\phi][\psi]\right)=$ $\bigcup\left(C_{f}[(\right.$ if $\left.\phi) \psi][\phi]\right)$, is equivalent to $\bigcup\left(C_{f}[\phi][\psi]\right)=\bigcup\left(C_{f}[\phi]\right)$. But since $C_{f}[\phi] \vDash$ $\psi$, this obtains. In the latter case $C_{f}[($ if $\phi) \psi][\phi][\psi]=\varnothing_{f}=C_{f}[($ if $\phi) \psi][\phi]$, so the entailment must also hold.

Definition 25 (Persistence) $\phi$ is persistent iff $C_{f}^{\prime} \vDash \phi$ if $C_{f} \vDash \phi$ and $\bigcup C^{\prime} \subseteq$ $\cup C$. (I.e. $\phi$ 's support persists after more information comes in.)

Fact 6 In general, $\diamond \phi$ is not persistent. Take a $c$ containing many worlds but only one $\phi$-world $w$. Then $\{c\}_{f} \vDash \diamond \phi$, but $c-\{w\} \subseteq c$ and $\{c-\{w\}\}_{f} \not \models \diamond \phi$. 
Fact 7 (Disj. Ants. 1) For persistent $\psi$, (if $\left.\phi_{1} \vee \phi_{2}\right) \psi \vDash\left(\right.$ if $\left.\phi_{1}\right) \psi \wedge$ (if $\left.\phi_{2}\right) \psi$ Proof The premise tests that $C_{f}\left[\phi_{1}\right] \cup C_{f}\left[\phi_{2}\right] \vDash \psi$. The conclusion presupposes that $\bigcup\left(C_{f}\left[\phi_{1}\right]\right) \neq \varnothing$ and $\bigcup\left(C_{f}\left[\phi_{2}\right]\right) \neq \varnothing$, and tests that $C_{f}\left[\phi_{1}\right] \vDash \psi$ and $C_{f}\left[\phi_{2}\right] \vDash \psi$. Since $\cup\left(C_{f}\left[\phi_{1}\right]\right) \subseteq\left(\cup\left(C_{f}\left[\phi_{1}\right]\right) \cup \cup\left(C_{f}\left[\phi_{2}\right]\right)\right)$ and $\cup\left(C_{f}\left[\phi_{2}\right]\right) \subseteq$ $\left(\cup\left(C_{f}\left[\phi_{1}\right]\right) \cup \cup\left(c\left[\phi_{2}\right]\right)\right)$, this test must be successful when $\psi$ is persistent but may not be successful when $\psi$ isn't persistent.

Remark 6 (if $p \vee \neg p) \diamond p \not \models(($ if $p) \diamond p) \wedge(($ if $\neg p) \diamond p)$. If there are both $p$ and $\neg \mathrm{p}$ worlds in $c$ all presuppositions will be met and the premise will successfully test $c$. The second conjunct of the conclusion won't.

Remark 7 While Fact 8 is limited to atomic $\alpha$ and $\beta$, it could be proven inductively for any $\alpha, \beta \in \mathcal{W} f f_{A}$, but I omit that lengthy proof here.

Fact 8 (Disj. Ants. 2) For persistent $\psi$ and atomic $\alpha, \beta$, (if $\triangleleft(\alpha \vee \beta)) \psi \vDash$ (if $\triangleleft \alpha) \psi \wedge($ if $\triangleleft \beta) \psi$

Proof The premise tests that $C_{f}[\triangleleft(\alpha \vee \beta)] \vDash \psi$. By Definition $24, C_{f}[\triangleleft(\alpha \vee$ $\beta)]=\left\{\left\{w^{\prime} \mid \exists w \in c: w^{\prime} \in f(w, a)\right\} \mid a \in\lfloor\alpha \vee \beta\rfloor\right\}_{f}$. By Definitions 18.4, 18.1

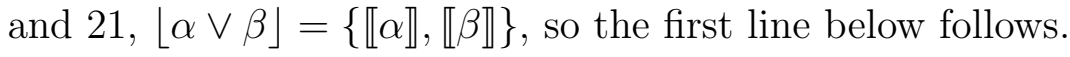

$$
\begin{aligned}
C_{f}[\triangleleft(\alpha \vee \beta)] & =\left\{\left\{w^{\prime} \mid \exists w \in c: w^{\prime} \in f(w, \llbracket \alpha \rrbracket)\right\},\left\{w^{\prime} \mid \exists w \in c: w^{\prime} \in f(w, \llbracket \beta \rrbracket)\right\}\right\}_{f} \\
& =\left\{\left\{w^{\prime} \mid \exists w \in c: w^{\prime} \in f(w, \llbracket \alpha \rrbracket)\right\}\right\}_{f} \cup\left\{\left\{w^{\prime} \mid \exists w \in c: w^{\prime} \in f(w, \llbracket \beta \rrbracket)\right\}\right\}_{f} \\
& =C_{f}[\triangleleft \alpha] \cup C_{f}[\triangleleft \beta] \\
& =C_{f}[\triangleleft \alpha \vee \triangleleft \beta]
\end{aligned}
$$

The second line follows by set theory, and the last two by Definitions 24 and 18.4, respectively. From this equality and Fact 7, the entailment follows.

\section{References}

Adams, E. W. (1975a). 'Counterfactual Conditionals and Prior Probabilities'. In Hooker, C. A. \& Harper, W., eds., 'Proceedings of International Congress on the Foundations of Statistics', Dordrecht, Holland: D. Reidel. Adams, E. W. (1975b). The Logic of Conditionals. Dordrecht: D. Reidel. Aloni, M. (2003). 'Free Choice in Modal Contexts'. In Weisgerber, M., ed., 'Proceedings of Sinn und Bedeutung 7', 25-37. Konstanz, Germany: Fachbereich Sprachwissenschaft der Universität Konstanz. URL http:// ling. uni-konstanz.de/pages/conferences/sub7/.

W. STARR

$03 / 23 / 13$ 
Alonso-Ovalle, L. (2006). Disjunction in Alternative Semantics. Ph.D. thesis, UMass Amherst, Amherst, MA.

Alonso-Ovalle, L. (2009). 'Counterfactuals, Correlatives, and Disjunction'. Linguistics and Philosophy 32(2): pp. 207-244. URL http://dx.doi.org/ 10.1007/s10988-009-9059-0.

Anderson, A. R. (1951). 'A Note on Subjunctive and Counterfactual Conditionals'. Analysis 12: pp. 35-38.

Arregui, A. (2007). 'When aspect matters: the case of would-conditionals'. Natural Language Semantics 15: pp. 221-264. URL http://dx.doi.org/ $10.1007 / \mathrm{s} 11050-007-9019-6$.

Arregui, A. (2009). 'On similarity in counterfactuals'. Linguistics and Philosophy 32: pp. 245-278. URL http://dx.doi.org/10.1007/ s10988-009-9060-7.

Bennett, J. (2003). A Philosophical Guide to Conditionals. Oxford: Oxford University Press.

van Benthem, J. (1986). Essays in Logical Semantics. Dordrecht: D. Reidel Publishing Co.

van Benthem, J., ed. (1991). Language in Action: Categories, Lambdas, and Dynamic Logic. Amsterdam: Elsevier Science.

van Benthem, J., Muskens, R. \& Visser, A. (2011). 'Dynamics'. In van Benthem, J. \& ter Meulen, A., eds., 'Handbook of Logic and Language', 607-670. Amsterdam: Elsevier Science Publishers.

Bittner, M. (2011). 'Time and Modality without Tenses or Modals'. In Musan, R. \& Rathers, M., eds., 'Tense Across Languages', 147188. Tübingen: Niemeyer. URL http://semanticsarchive.net/Archive/ zliYmQxY/bittner11_tam.pdf.

Brasoveanu, A. (2010). 'Decomposing Modal Quantification'. Journal of Semantics 27(4): pp. 437-527. URL http://jos.oxfordjournals.org/ content/27/4/437. abstract.

Briggs, R. (2012). 'Interventionist Counterfactuals'. Philosophical Studies 160(1): pp. 139-166.

Clark, H. H. (1996). Using Language. Cambridge, England: Cambridge University Press.

Dekker, P. (2012). Dynamic Semantics, vol. 91 of Studies in Linguistics and Philosophy. Dordrecht: Springer Netherlands.

Dudman, V. C. (1981). 'Time and Tense in English'. Unpublished manuscript, Macquarie University.

Dudman, V. C. (1983). 'Tense and Time in English Verb CLusters of the

W. Starr

$03 / 23 / 13$

$5^{6}$ 
Primary Pattern'. Australasian Journal of Linguistics 3: pp. 25-44.

Dudman, V. H. (1984). 'Parsing 'If'-Sentences'. Analysis 44(4): pp. 145153. http://analysis.oxfordjournals org/content/44/4/145. full. pdf+html, URL http://analysis.oxfordjournals.org/content/44/4/ 145. short.

Dudman, V. H. (1988). 'Indicative and Subjunctive'. Analysis 48(3): pp. 113-122. http://analysis.oxfordjournals.org/content/48/3/113.2. full.pdf+html, URL http://analysis.oxfordjournals.org/content/ 48/3/113.2. short.

Ellis, B., Jackson, F. \& Pargetter, R. (1977). 'An Objection to Possible-World Semantics for Counterfactual Logics'. Journal of Philosophical Logic 6(3): pp. 355-357.

Fagin, R., Halpern, J. Y., Moses, Y. \& Vardi, M. Y. (1995). Reasoning about Knowledge. Cambridge, Massachusetts: The MIT Press.

Fine, K. (1975). 'Review of Lewis' Counterfactuals'. Mind 84: pp. 451-8.

Fine, K. (2012a). 'Counterfactuals Without Possible Worlds'. Journal of Philosophy 109(3): pp. 221-246.

Fine, K. (2012b). 'A Difficulty for the Possible Worlds Analysis of Counterfactuals'. Synthese 189(1): pp. 29-57.

VON Fintel, K. (1999a). 'NPI Licensing, Strawson Entailment Context Dependency'. Journal of Semantics 16: pp. 97-148.

von Fintel, K. (1999b). 'The Presupposition of Subjunctive Conditionals'. In Sauerland, U. \& Percus, O., eds., 'The Interpretive Tract', vol. MIT Working Papers in Linguistics 25, 29-44. Cambridge, MA: MITWPL. URL http://mit.edu/fintel/www/subjunctive.pdf.

von Fintel, K. (2001). 'Counterfactuals in a Dynamic Context'. In Kenstowicz, M., ed., 'Ken Hale: a Life in Language', 123-152. Cambridge, Massachusetts: The MIT Press. URL http://mit.edu/fintel/ www/conditional.pdf.

von Fintel, K. (2012). 'Subjunctive Conditionals'. In Russell, G. \& FARA, D. G., eds., 'The Routledge Companion to Philosophy of Language', 466-477. New York: Routledge. URL http://mit.edu/fintel/ fintel-2012-subjunctives.pdf.

von Fintel, K. \& Gillies, A. S. (2011). 'Might Made Right'. In Egan, A. \& Weatherson, B., eds., 'Epistemic Modality', 108-130. Oxford University Press. URL http://semanticsarchive.net/Archive/TAyZGY2Z/ mmr.pdf.

Frank, A. \& Kamp, H. (1997). 'On Context Dependence in Modal Con-

W. STARR

$03 / 23 / 13$ 
structions'. In Lawson, A., ed., 'Proceedings from Semantics and Linguistic Theory VII', 151-168. Ithaca, NY: Cornell University.

Gärdenfors, P. (1982). 'Imaging and Conditionalization'. Journal of Philosophy 79(12): pp. 747-760.

Gillies, A. (2004). 'Epistemic Conditionals and Conditional Epistemics'. Noûs 38(4): pp. 585-616. URL http://rci.rutgers.edu/ thony/epi_ cond_nous.pdf.

Gillies, A. (2007). 'Counterfactual Scorekeeping'. Linguistics 83 Philosophy 30(3): pp. 329-360. URL http://rci.rutgers.edu/ thony/ counterfactualscorekeeping_landp.pdf.

Gillies, A. (2009). 'On Truth-Conditions for If (but Not Quite Only If)'. Philosophical Review 118(3): pp. 325-349. URL http://rci.rutgers. edu/ thony/truth-conditions-if-phil-review-2009.pdf.

Goodman, N. (1947). 'The Problem of Counterfactual Conditionals'. The Journal of Philosophy 44: pp. 113-118.

Groenendisk, J., Stokhof, M. \& Veltman, F. (1996). 'Coreference and Modality'. In LAPPIN, S., ed., 'The Handbook of Contemporary Semantic Theory', 179-213. Oxford: Blackwell Publishers.

Heim, I. (1982). The Semantics of Definite and Indefinite Noun Phrases. Ph.D. thesis, Linguistics Department, University of Massachusetts, Amherst, Massachusetts.

Herzberger, H. (1979). 'Counterfactuals and Consistency'. Journal of Philosophy 76(2): pp. 83-88.

Hobbs, J., Stickel, M., Appelt, D. \& Martin, P. (1993). 'Interpretation as Abduction'. Artificial Intelligence 63(1-2): pp. 69-142.

IATRIDOU, S. (2000). 'The Grammatical Ingredients of Counterfactuality'. Linguistic Inquiry 31(2): pp. 231-270.

IpPolito, M. (2003). 'Presuppositions and Implicatures in Counterfactuals'. Natural Language Semantics 11(2): pp. 145-186.

IsARD, S. (1974). 'What Would You Have Done If...' Theoretical Linguistics 1: pp. 233-55.

Kamp, H. (1981). 'A Theory of Truth and Semantic Representation'. In GroenendiJk, J. A., Janssen, T. \& Stokhof, M., eds., 'Formal Methods in the Study of Language', 277-322. Dordrecht: Foris.

Katsuno, H. \& Mendelzon, A. (1992). 'On the Difference Between Updating a Knowledge Base and Revising It'. In Gärdenfors, P., ed., 'Belief Revision', 183-203. Cambridge: Cambridge University Press.

Kaufmann, S. (2000). 'Dynamic Context Management'. In Faller, M.,

W. StARR

$03 / 23 / 13$

$5^{8}$ 
Kaufmann, S. \& Pauly, M., eds., 'Formalizing the Dynamics of Conversation', 171-188. Stanford, CA: CSLI Publications.

Kaufmann, S. (2005). 'Conditional Truth and Future Reference'. Journal of Semantics 22(3): pp. 231-280. URL http://jos . oxfordjournals .org/ content/22/3/231. abstract.

Kratzer, A. (1989). 'An Investigation of the Lumps of Thought'. Linguistics and Philosophy 12(5): pp. 607-653.

LeAHY, B. (2011). 'Presuppositions and Antipresuppositions in Conditionals'. In Ashton, N., Chereches, A. \& Lutz, D., eds., 'Proceedings from Semantics and Linguistic Theory (SALT) 21', 257-274.

Lewis, C. I. (1914). 'The Calculus of Strict Implication'. Mind 23(90): pp. 240-247. URL http://www. jstor.org/stable/2248841.

Lewis, D. K. (1969). Convention: A Philosophical Study. Cambridge, MA: Harvard University Press.

Lewis, D. K. (1973). Counterfactuals. Cambridge, Massachusetts: Harvard University Press.

Lewis, D. K. (1975). 'Languages and Language'. In Gunderson, K., ed., 'Language, Mind, and Knowledge. Minnesota Studies in the Philosophy of Science, Vol. 7', 3-35. Minneapolis, Minnesota: University of Minnesota Press.

Lewis, D. K. (1979a). 'Counterfactual Dependence and Time's Arrow'. Noûs 13: pp. 455-476.

LEWIS, D. K. (1979b). 'Scorekeeping in a Language Game'. Journal of Philosophical Logic 8(1): pp. 339-359. URL http://www.jstor.org/stable/ 30227173.

LEWIS, K. S. (2012). 'Discourse dynamics, pragmatics, and indefinites'. Philosophical Studies 158: pp. 313-342. URL http://dx.doi.org/10.1007/ s11098-012-9882-y.

Loewer, B. (1976). 'Counterfactuals with Disjunctive Antecedents'. Journal of Philosophy 73(16): pp. 531-537.

LyCAN, W. G. (2001). Real Conditionals. Oxford: Oxford University Press.

Lyons, J. (1977). Semantics, vol. 2. Cambridge, England: Cambridge University Press.

McCarthy, J. \& Hayes, P. J. (1969). 'Some Philosophical Problems from the Standpoint of Artificial Intelligence'. In Meltzer, B. \& Michie, D., eds., 'Machine Intelligence 4', 463-502. Edinburgh: Edinburgh University Press.

McGee, V. (1985). 'A Counterexample to Modus Ponens'. Journal of Phi-

W. Starr

$03 / 23 / 13$ 
losophy 82: pp. 462-471.

McGee, V. (1989). 'Conditional Probabilities and Compounds of Conditionals'. Philosophical Review 98(4): pp. 485-541.

McKay, T. J. \& van Inwagen, P. (1977). 'Counterfactuals with Disjunctive Antecedents'. Philosophical Studies 31: pp. 353-356.

Montague, R. (1970). 'Pragmatics and Intensional Logic'. Synthese 22(1/2): pp. pp. 68-94. URL http://www.jstor.org/stable/20114750.

Moss, S. (to appear). 'On the Pragmatics of Counterfactuals'. Noûs URL http://www-personal umich. edu/ ssmoss/counterfactuals.pdf.

Nolan, D. (2003). 'Defending a Possible-Worlds Account of Indicative Conditionals'. Philosophical Studies 116(3): pp. 215-269.

Nute, D. (1975). 'Counterfactuals and the Similarity of Worlds'. The Journal of Philosophy 72(21): pp. 773-8.

Nute, D. (1980). 'Conversational Scorekeeping and Conditionals'. Journal of Philosophical Logic 9: pp. 153-166.

Pearl, J. (1996). 'Causation, Action, and Counterfactuals'. In Shoham, Y., ed., 'Theoretical Aspects of Rationality and Knowledge: Proceedings of the Sixth Conference (TARK 1996)', 51-73. San Francisco: Morgan Kaufmann.

Pearl, J. (2000). Causality: Models, Reasoning, and Inference. Cambridge, England: Cambridge University Press.

Pollock, J. (1976). 'The 'Possible Worlds' Analysis of Counterfactuals'. Philosophical Studies 29: pp. 469-476.

Roberts, C. (1989). 'Modal Subordination and Pronominal Anaphora in Discourse'. Linguistics and Philosophy 12(6): pp. 683-721.

van Rooy, R. (2006). Attitudes and Changing Contexts. Dordrecht: Springer. Rothschild, D. \& Yalcin, S. (2013). 'On the Dynamics of Conversation'. Ms. Oxford University and UC Berkeley, URL http://philpapers.org/ rec/ROTOTD.

Schulz, K. (2007). Minimal Models in Semantics and Pragmatics: free choice, exhaustivity, and conditionals. Ph.D. thesis, University of Amsterdam: Institute for Logic, Language and Information, Amsterdam. URL http://www . illc.uva.nl/Publications/Dissertations/DS-2007-04.text.pdf.

Shanahan, M. (2009). 'The Frame Problem'. In Zalta, E. N., ed., 'The Stanford Encyclopedia of Philosophy', Winter 2009 edn. URL http: //plato.stanford.edu/archives/win2009/entries/frame-problem/.

Simons, M. (2005). 'Dividing Things Up: The Semantics of or and the Modal/or Interaction'. Natural Language Semantics 13(3): pp. 271-316.

Skyrms, B. (1974). 'Contraposition of the Conditional'. Philosophical Studies

W. Starr

$03 / 23 / 13$

60 
26(2): pp. 145-147.

Stalnaker, R. C. (1968). 'A Theory of Conditionals'. In Rescher, N., ed., 'Studies in Logical Theory', 98-112. Oxford: Basil Blackwell Publishers.

Stalnaker, R. C. (1975). 'Indicative Conditionals'. Philosophia 5: pp. 269-286. Page references to reprint in Stalnaker (1999).

Stalnaker, R. C. (1978). 'Assertion'. In Cole, P., ed., 'Syntax and Semantics 9: Pragmatics', 315-332. New York: Academic Press. References to reprint in Stalnaker (1999).

Stalnaker, R. C. (1984). Inquiry. Cambridge, Massachusetts: The MIT Press.

Stalnaker, R. C. (1998). 'On the Representation of Context'. Journal of Logic, Language, and Information 7(1): pp. 3-19.

Stalnaker, R. C. (1999). Context and Content: Essays on Intentionality in Speech and Thought. Oxford: Oxford University Press.

Stalnaker, R. C. (2002). 'Common Ground'. Linguistics and Philosophy 25(5-6): pp. 701-721.

Stalnaker, R. C. (2005). 'Conditional Propositions and Conditional Assertions'. In Gajewski, J., Hacquard, V., Nickel, B. \& Yalcin, S., eds., 'New Work on Modality', vol. 51 of MIT Working Papers in Linguistics. Cambridge, MA: MITWPL.

Starr, W. B. (2010). Conditionals, Meaning and Mood. Ph.D. thesis, Rutgers University, New Brunswick, NJ. URL http://hdl.rutgers.edu/ 1782.1/rucore10001600001.ETD.000056780.

Starr, W. B. (2011). 'What If?' Ms. Cornell University.

StarR, W. B. (2012). 'A Uniform Theory of Conditionals'. Ms. Cornell University.

Starr, W. B. (2013a). 'Indicative Conditionals: Logic, Truth and Pragmatics'. Ms. Cornell University.

Starr, W. B. (2013b). 'Structured Possible Worlds'. Ms. Cornell University.

Stone, M. (1997). 'The Anaphoric Parallel between Modality and Tense'. Tech. Rep. 97-06, University of Pennsylvania Institute for Research in Cognitive Science, Philadelphia, PA. URL http://www.cs.rutgers.edu/ $\sim$ mdstone/pubs/ircs97-06.pdf.

Stone, M. (1999). 'Reference to Possible Worlds'. Tech. Rep. 49, Rutgers University Center for Cognitive Science. Updated version of Stone (1997), URL http://www.cs.rutgers . edu/ mdstone/pubs/ruccs-49.pdf.

Thomason, R. H. (2007). 'Conditionals and Action Logics'. In 'Logical Formalizations of Commonsense Reasoning: papers from the AAAI Spring

W. STARR

$03 / 23 / 13$ 
Symposium', AAAI Technical Report SS-07-05, Menlo Park, CA: AAAI Press.

Van linden, A. \& Verstraete, J.-C. (2008). 'The nature and origins of counterfactuality in simple clauses: Cross-linguistic evidence'. Journal of Pragmatics 40(11): pp. 1865 - 1895. URL http://www.sciencedirect. com/science/article/pii/S0378216608000647.

Veltman, F. (1985). Logics for Conditionals. Ph.D. dissertation, University of Amsterdam, Amsterdam.

Veltman, F. (1986). 'Data Semantics and the Pragmatics of Indicative Conditionals'. In Traugott, E., ter Meulen, A., Reilly, J. \& Ferguson, C., eds., 'On Conditionals', Cambridge, England: Cambridge University Press.

Veltman, F. (1996). 'Defaults in Update Semantics'. Journal of Philosophical Logic 25(3): pp. 221-261. URL http://dx. doi .org/10.1007/BF00248150.

Veltman, F. (2005). 'Making Counterfactual Assumptions'. Journal of Semantics 22: pp. 159-180. URL http://staff . science.uva.nl/ veltman/ papers/FVeltman-mca.pdf.

WARmBrōD, K. (1981a). 'Counterfactuals and Substitution of Equivalent Antecedents'. Journal of Philosophical Logic 10(2): pp. 267-289.

WarmBrōD, K. (1981b). 'An Indexical Theory of Conditionals'. Dialogue, Canadian Philosophical Review 20(4): pp. 644-664.

WarmbrōD, K. (1982). 'A Defense of the Limit Assumption'. Philosophical Studies 42(1): pp. 53-66.

WarmbrōD, K. (1983). 'Epistemic Conditionals'. Pacific Philosophical Quarterly 64: pp. 249-265. URL http://philpapers.org/archive/WAREC.1. pdf.

Williams, J. R. G. (2008). 'Conversation and Conditionals'. Philosophical Studies 128: pp. 211-233. URL http://www.personal.leeds.ac.uk/ \%7Ephljrgw/wip/conversationconditionals.pdf. 